\title{
Connectedness and part-relation integration in shape category learning
}

\author{
JUN SAIKI \\ Nagoya University, Nagoya, Japan \\ and \\ JOHN E. HUMMEL \\ University of Califormia, Los Angeles, Califormia
}

\begin{abstract}
We investigated the role of connectedness in the use of part-relation conjunctions for object category learning. Participants learned categories of two-part objects defined by the shape of one part and its location relative to the other (part-relation conjunctions). The topological relationship between the parts (connected, separated, or embedded) varied between participants but was invariant for any given participant. In Experiment 1, category learning was faster and more accurate when an object's parts were connected than when they were either separated or embedded. Subsequent experiments showed that this effect is not due to conscious strategies, differences in the salience of the individual attributes, or differences in the integrality/separability of dimensions across stimuli. The results suggest that connectedness affects the integration of parts with their relations in object category learning.
\end{abstract}

Shape plays a central role in object categorization (Rosch, Mervis, Gray, Johnson, \& Boyes-Braem, 1976; see also Biederman, 1987, and Saiki \& Hummel, 1996). The mental representation of shape therefore imposes important constraints on object categorization and category learning. Object shape cannot be described as a single feature or as a value on any single dimension. Rather, most objects have complex shapes composed of many simpler shapes (parts or features) in specific spatial relations to one another (Biederman, 1987; Marr \& Nishihara, 1978). As a result, object perception and categorization entail more than simply detecting an object's features and matching them to memory; it is also necessary to integrate (or bind) an object's parts with their relations to form a coherent description of the object's shape (Hummel \& Biederman, 1992; Saiki \& Hummel, 1996; see also Barsalou, 1993, and Hummel \& Stankiewicz, 1996). For example, consider the act of perceiving and categorizing a chair. A chair is not simply a collection of vertical posts (the legs), a horizontal slab (the seat) and a vertical slab (the back). To form a chair, these parts must be in particular spatial relations. Distinguishing a chair from a ran-

This research was supported by a research grant to J.E.H. from the UCLA Academic Senate. The authors are grateful to Brian Stankiewicz for his extremely helpful suggestions during the development of this project. The authors also thank Barbara Malt, Gregory Lockhead, Robert Nosofsky, and two anonymous reviewers for helpful comments on an earlier draft of this manuscript. Correspondence should be addressed to J. Saiki, Graduate School of Human Informatics, Nagoya University, Furoh-cho, Chikusa-ku, Nagoya, Japan 464-01 (e-mail: saiki@info.human.nagoya-u.ac.jp).

- Accepted by previous associate editor Peter Dixon dom collection of chair parts entails both perceiving the parts and their spatial relations and binding the parts to the appropriate relations (Hummel \& Biederman, 1992; see also Fodor \& Pylyshyn, 1988). For example, a structure consisting of a seat supporting four legs contains the same parts and relations as four legs supporting a seat; these objects (only one of which is a chair) differ only in their part-relation bindings (i.e., which parts stand in which role of the support relation).

Part-relation binding is not a trivial perceptual problem. If two parts belong to the same object, then it is sensible to integrate them with their interrelations and incorporate the resulting part-relation bindings into the object's structural description. However, most images contain multiple objects, and it is not sensible to integrate the parts of one object into the structural description of another. Given a pair of parts in an image, the visual system must decide whether those parts belong to the same object. If they do, then it should integrate them with their interrelations; if they do not, it should ignore their relations (at least for the purposes of object recognition; see Saiki \& Hummel, 1998). This problem is a manifestation of the familiar figure-ground segregation problem, and it must be solved prior to identifying the objects (Saiki \& Hummel, 1998; but see Peterson \& Gibson, 1994).

As detailed shortly, we recently reported results supporting the role of explicit part-relation integration in shape perception (Saiki \& Hummel, 1998). We have also shown that part-relation bindings play a privileged role in object category learning (Saiki \& Hummel, 1996). The present experiments investigated whether factors that affect part-relation integration in shape perception also affect part-relation integration in object category learning 
(i.e., in the long-term memory representation of object shape). In contrast to object perception, which only requires the visual system to construct a representation of an object's shape, recognition and categorization require the system both to construct a representation of the object's shape (perception) and to match that representation to one or more representations in memory (recognition/ categorization). Factors affecting how object categories are represented in memory are therefore just as important to object categorization as factors affecting how those shapes are perceived. Visual cues suggesting that two parts are physically connected (in the physical world) affect part-relation integration in shape perception (Saiki \& Hummel, 1998). The present study investigated whether such cues also affect the integration of parts with their relations in object memory: Does connectedness affect the formation of categories (i.e., equivalence classes) in long-term memory? If it does, then this finding would show that a perceptual constraint on part-relation integration (i.e., connectedness) also affects the cognitive process of category learning.

In this paper, we will use the term connectedness to refer to perceptual cues (i.e., properties of the proximal stimulus) suggesting the physical connectedness of parts in the distal stimulus (i.e., world). As elaborated shortly, connectedness in a visual image (i.e., when two figures touch, occupying overlapping regions in the image) does not necessarily imply connectedness in the distal stimulus. For example, one object can occlude another in the image even if the two do not touch in the three-dimensional (3-D) world.

\section{Part-Relation Integration in \\ Object Perception and Categorization}

Most scenes contain many objects, each composed of multiple parts. For the purposes of object recognition, it is necessary to group the parts in an image into objectbased sets (to decide which parts to consider together as parts of the same object). Connectedness is a strong cue for perceptual grouping that may serve this function (Palmer, 1992; Palmer \& Rock, 1994; Saiki \& Hummel, 1998): Two parts (e.g., geons) are more likely to be connected in a visual image if they belong to the same object (and are therefore connected in the 3-D world) than if they belong to different objects. Consistent with this idea, we showed that part connectedness (in the image) facilitates part-relation integration in shape perception (Saiki \& Hummel, 1998). Using rapid serial visual presentation (RSVP), we found that people were better able to detect targets defined by part-relation conjunctions when the parts were connected than when they were spatially separated. These findings are part of a larger literature showing that the relations among features (or parts) of the same object are processed differently than the relations among features of separate objects. For example, Baylis and Driver (1993) showed that people are faster to judge the relative height of two vertices when they belong to the same object than when they belong to different ob- jects, and Elder and Zucker (1993) showed that closed figures are easier to detect in a visual search task than are open figures.

These and related findings (e.g., Donnelley, Humphreys, \& Riddoch, 1991; Enns, 1992) suggest that within-object relations (relations between features or parts of the same object) are represented and/or processed differently than between-object relations (relations between features or parts of different objects; see Saiki \& Hummel, 1998, for a review). However, all these effects were observed using perceptual tests and, except for the visual search tasks of Donnelley et al. (1991) and Enns (1992), speeded stimulus presentations. It is therefore possible that the effects of perceptual integration are strictly perceptual, affecting the ease with which a shape will be perceived, but (given that it is perceived) not the manner in which it is encoded into memory. This account is intuitively appealing: It does not "feel" as though our memory for one of Elder and Zucker's (1993) closed figures is any better or more veridical than our memory for one of their open figures, and there are no a priori grounds for assuming that categories of the former type would be any easier to learn than categories of the latter. According to this intuitive account, factors affecting perceptual integration should influence category learning only to the extent that they interfere with one's ability to perceive the to-be-categorized stimuli. For example, their effects should be negligible if subjects are permitted to view the stimuli long enough to perceptually integrate their features and relations. Alternatively, it is possible that cues affecting perceptual integration affect not only the ease with which a relation is perceived but also the manner in which it is treated as a candidate for category learning. If this is the case, then such cues may influence object category learning even when subjects are given unlimited time to view the stimuli.

What reason is there to expect a cue for perceptual integration (such as connectedness) to influence unspeeded category learning, and what form might such an effect take? If object categories are structured in the sense of specifying part-relation bindings, then, for the purposes of categorization, those bindings must not only be perceived in the stimulus but must also be encoded into object memory (Hummel \& Biederman, 1992; Hummel \& Saiki, 1993). (If the bindings are not encoded during category learning, then they can be of no use in subsequent object categorization, even if they are plainly visible in the stimulus.) If connectedness affects the likelihood that a part-relation binding will be encoded into memory, then it could also affect category learning, even if the parts and their relations are plainly visible.

We recently reported an effect not unlike this. Participants in the location condition of Saiki and Hummel (1996, Experiments 1, 2, and 4) learned to categorize objects defined by conjunctions of parts in particular spatial relations. For example, a typical member of the category Hoz might consist of a cone-shaped part on the right-hand end of a brick-shaped part, and a typical $J i k$ might consist of a curved cylinder centered on the top of 


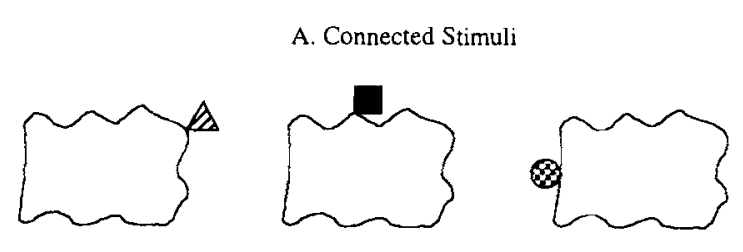

B. Separated Stimuli

e
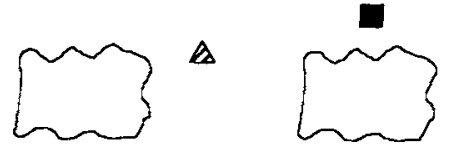

8

C. Embedded Stimuli
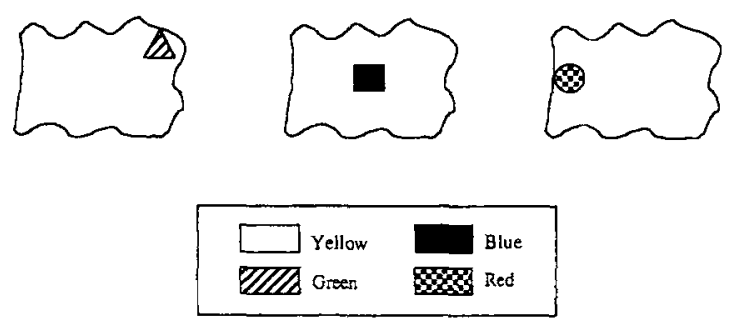

Figure 1. Examples of the stimuli used in Experiment 1.

a brick. Hoz is thus defined by the conjunction cone + right end, whereas Jik is defined by curved cylinder + top-center. The conjunctions are critical. Objects with a curved cylinder (the part shape typical of Jik) on the right end of the brick (the relation typical of $\mathrm{Hoz}$ ) belonged to neither Hoz nor Jik. As in the chair example discussed previously, this kind of learning situation is typical of natural object category learning: Most objects are defined by the presence of particular parts in particular relations to one another (see Saiki \& Hummel, 1996).

The other condition in these experiments was the color condition, in which categories were defined by conjunctions of part shapes and colors. For example, a typical $\mathrm{Hoz}$ might have a red cone somewhere on a brick (the conjunction cone-shaped + red $)$, and a typical Jik might have a blue cylinder on a brick (the conjunction cylindershaped + blue). In both cases, the spatial relation between the cone or cylinder and the brick was irrelevant and free to vary. Except for replacing the variable relative location with the variable color, this condition was logically identical to the location condition. But, in spite of their having identical logical structures, participants in the location condition learned the object categories with much greater ease (i.e., much faster, and to a higher level of accuracy) than did participants in the color condition. Importantly, this effect obtained even though learning categories based on colors alone was just as easy as learning colors based on relative location alone (Saiki \& Hummel, 1996, Experiment 1).
We interpreted the difference between part-relation and part-color category learning as reflecting a bias to learn object categories based on part-relation conjunctions rather than on part-color conjunctions. This differential sensitivity affected object category learning even though the part-color conjunctions were undoubtedly visible in the stimuli. In turn, this suggests that the differential sensitivity manifested itself specifically in the encoding of the bindings into long-term memory, rather than the perception of those bindings. The experiments reported here were designed to test the hypothesis that connectedness can create analogous effects in object category learning: Will participants find it easier to learn object categories defined by part-relation conjunctions when the objects' parts are connected than when they are separated or embedded (as defined below)? If so, then this effect would suggest that connectedness, which is known to affect part-relation binding in shape perception (Saiki \& Hummel, 1998), can also affect part-relation binding in object memory.

\section{Overview of the Present Study}

This paper reports a series of experiments exploring the effects of connectedness on part-relation integration in category learning. Participants learned to categorize two-part "objects," where object categories were defined by the shape of one part (a "variable" part, so named because its shape was free to vary) and its position relative to a second part (the "common" part, so named because its shape remained constant across all stimuli). Our objects were not object-like in the usual sense of the word (as revealed in Figure 1), but, for the sake of clarity, we shall refer to them as objects. For one group of participants, the variable and common parts were connected, in that the bounding contour of the variable part touched the bounding contour of the common part (Figure 1). For a second group, the variable and common parts were spatially separated. For a third group, the bounding contour of the variable part was contained completely within the bounding contour of the common part (Figure 1). The parts and relations that defined the object categories were otherwise identical across groups of participants.

We distinguish embeddedness from connectedness because we assume that connectedness (as defined in our stimuli) is a strong visual cue suggesting physical connectedness, whereas embeddedness (as defined in our stimuli) is a much weaker cue to physical connectedness. This assumption is motivated by Lowe's (1987) "nonaccidentalness" constraint: The bounding contours of physically separated parts are unlikely to touch (as they do in our connected stimuli) just by an accident of viewpoint. (Note that physically separate objects occluding one another typically overlap over a large area of an image, rather than meeting exactly at a point or line.) This type of connectedness thus serves as a strong perceptual cue for physical connectedness in the 3-D world. In contrast, embeddedness-where the image of one object is contained wholly inside the image of another-is much more likely 
Table 1

Category Structure of Experiments 1 and 2

\begin{tabular}{|c|c|c|c|c|c|}
\hline \multirow[b]{2}{*}{$\begin{array}{c}\text { Exemplar } \\
\text { Type }\end{array}$} & \multirow[b]{2}{*}{ Frequency } & \multicolumn{4}{|c|}{ Attributes } \\
\hline & & Shape & $\begin{array}{l}\text { Relative } \\
\text { Location }\end{array}$ & Color & $\begin{array}{l}\text { Absolute } \\
\text { Location }\end{array}$ \\
\hline$h h$ & 7 & $h$ & $h$ & $r$ & $r$ \\
\hline$h n$ & 1 & $h$ & $n$ & $\mathrm{r}$ & $\mathrm{r}$ \\
\hline$n h$ & 1 & $n$ & $h$ & $r$ & $r$ \\
\hline$j j$ & 7 & $j$ & $j$ & $r$ & $\mathrm{r}$ \\
\hline jn & 1 & $j$ & $n$ & $r$ & $r$ \\
\hline$n j$ & 1 & $n$ & $j$ & $\mathrm{r}$ & $r$ \\
\hline$n n$ & 3 & $n$ & $n$ & $\mathrm{r}$ & $\mathrm{r}$ \\
\hline$h j$ & 3 & $h$ & $j$ & $\mathrm{r}$ & $r$ \\
\hline jh & 3 & $j$ & $h$ & $\mathrm{r}$ & $r$ \\
\hline
\end{tabular}

to happen just by chance and is thus a much weaker cue to physical connectedness. Embedded figures are more likely to be interpreted as figure and ground (e.g., Baylis \& Driver, 1995) or as a part with a texture element within it. At the same time, however, embeddedness shares with connectedness the property of spatial proximity: Embedded parts, like connected parts, are close together. (Indeed, embedded figures are arguably closer together than are connected figures.) Defining and manipulating connectedness and embeddedness in this fashion permits us to separate the effects of connectedness from the effects of simple spatial proximity.

If connectedness affects part-relation integration in object category learning, then participants in the connected condition should learn the categories faster (and/or to a higher level of accuracy) than should participants in the separated and embedded conditions. This task differed from a typical perceptual identification task (such as the RSVP task of our previous study) in that participants were given unlimited time to view the objects. Under these conditions, it is certainly possible to perceive the relations between separated parts and to perceptually bind the relations to the parts to which they refer (as revealed in Figure 1). If connectedness affects category learning under these conditions, then this will suggest that the role of connectedness in part-relation integration goes beyond simply facilitating perceptual binding.

What would it mean for connectedness to affect partrelation binding in the long-term memory representation of an object category? Recall that categorization entails matching the perceptual representation of a stimulus to the long-term memory representation of the category. A given part-relation binding can influence categorization only if it is part of the memory representation of the category (or of a "competing" category). For example, consider Hummel and Biederman's (1992) JIM model of object recognition. In this model, the long-term memory representation of an object includes units that respond specifically to conjunctions of parts and their interrelations. For instance, the memory representation of an object composed of a cone on top of a brick has one unit that responds to CONE-SHAPED +ON-TOP-OF and another unit that responds to ERICK-SHAPED + BELOW. These units jointly activate the object unit for CONE-ON-TOP-OF-BRICK. The conjunction units CONE-SHAPED +ON-TOP-OF and BRICK-SHAPED + BELOW code the part-relation bindings in long-term memory. Without them (e.g., if units for CONE, BRICK, ON-TOP, and BELOW directly activated the unit for CONE-ON-TOP-OF-BRICK), the model would incorrectly categorize a brick on top of a cone as a member of the class cones-on-top-of-bricks (Hummel \& Biederman, 1992). The experiments reported here were designed to investigate whether part connectedness influences the encoding of such part-relation conjunctions in the memory representation of an object category.

It is important to emphasize at this point that our primary interest concerns the role of part-relation integration (i.e., the use of part-relation bindings) in the formation of object equivalence classes (categories); we are not currently interested in participants' episodic representations of the individual objects. A category learning task is more appropriate for our purposes than an explicit memory task.

\section{EXPERIMENT 1}

Experiment 1 was designed to test our primary hypothesis that connectedness will affect participants' ability to learn categories defined by conjunctions of part shape and relative location. Following Saiki and Hummel (1996), we used a nonexhaustive two-category structure (illustrated in Table 1). All objects had two parts. Object categories were defined by a conjunction of two attributes: the shape of one part (the variable part) and its location relative to the other part (the common part). For example, the category $\mathrm{Hoz}$ might consist of objects with a small circle (variable part) to the left of a large wiggly rectangle (common part): Hoz is defined by the conjunction circle and left-of. The category Jik might consist of objects with a small square on top of the rectangle (square and ontop). An object with a circle on top of the rectangle and one with a square on the left belong to neither Hoz nor Jik. The correct response to such objects is "Neither."

Performance on the Neither category provides an index of participants' ability to categorize objects on the basis of conjunctions of part shape and relative location (Saiki \& 




Exemplar hh

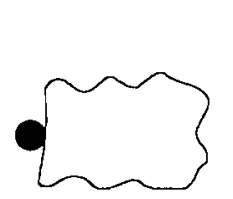

Exemplar jj

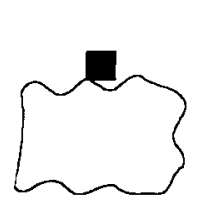

Exemplar nn

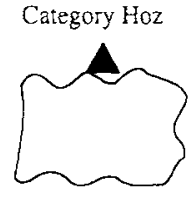

Exemplar hn

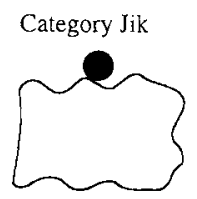

Exemplar jn

Category Neither

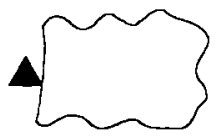

Exemplar hj

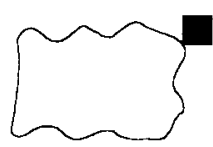

Exemplar nh

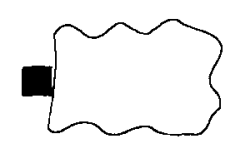

Exemplar nj

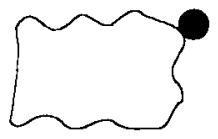

Exemplar jh
Yellow

Blue

Figure 2. Examples of the nine exemplar types in the connected condition. Names of exemplar types correspond to those denoted in Table 1. Colors are denoted by textures, and, for simplicity, only one color is shown (in the experiments, there were three values each, randomly presented). The separated and embedded exemplars were generated in an analogous fashion.

Hummel, 1996). People who classify objects on the basis of part-relation conjunctions will correctly classify members of the Neither category; however, those who classify objects on the basis of part shape or relative location alone will tend to misclassify Neither objects as members of Hoz or Jik. Performance with the Neither category was therefore the critical dependent variable in this experiment. If connectedness plays a role in classification based on part-relation conjunctions, then participants in the connected condition were expected to correctly classify Neither objects more often than participants in the separated and embedded conditions. As noted previously, performance in the embedded condition served to distinguish the role of proximity from the role of connectedness per se.

\section{Method}

Participants. Thirty-six undergraduates at the University of California, Los Angeles (UCLA), participated for credit in introductory psychology classes. All had normal color vision. The participants were randomly assigned to experimental conditions.

Design. There was one between-subjects independent variable, stimulus set, with three conditions: connected, separated, and embedded.

Stimuli. Stimuli were colored line drawings of 2-D objects (Figure 1). Each object was composed of two parts: The common part was a wavy yellow rectangle, which was common to all stimuli; the variable part was smaller than the common part, and its shape, color, and location relative to the common part varied across stimuli. In the connected and embedded stimulus sets, the common part was $8 \mathrm{~cm}$ high and $12 \mathrm{~cm}$ wide, and the variable parts were approximately $2 \mathrm{~cm}$ high and $2 \mathrm{~cm}$ wide. In the separated set, both the common and the variable parts were reduced to $80 \%$ of their size in the connected/embedded sets, making the common part $6.4 \times$ $9.6 \mathrm{~cm}$ and the variable parts approximately $1.6 \times 1.6 \mathrm{~cm}$. The participants sat approximately $80 \mathrm{~cm}$ from the display, so the common and variable parts subtended between $6^{\circ} \times 9^{\circ}$ (connected and embedded) and $4.8^{\circ} \times 7.2^{\circ}$ (separated) and between $1.5^{\circ} \times 1.5^{\circ}$ (connected and embedded) and $1.2^{\circ} \times 1.2^{\circ}$ (separated), respectively, Stimuli varied in the shape of the variable part (circle, square, or triangle), the color of the variable part (red, blue, or green), the location of the variable part relative to the common part (explained shortly), and the absolute location of the whole object on the computer screen (upper left, lower center, or middle right).

There were three stimulus sets. In the connected set (Figure 1A), the two parts of each stimulus were connected as defined above. The locations of the variable part in these stimuli were (1) at the top and center of the common part, (2) at the middle of the left side of the common part, and (3) at the upper right corner of the common part. Each object in the separated set was created by moving the variable part of one object in the connected set so that it no longer touched the common part (Figure 1B). This procedure was adopted to ensure that the separated objects would be analogous to the connected objects in terms of the relative locations of their parts. Because separating their parts makes a separated object larger, on the whole, than the corresponding connected object, the separated objects were reduced in size as described previously. Each object in the embedded set (Figure 1C) was created by moving the variable part of one connected object inside the common part, again keeping the relations in the embedded set analogous to those in the connected set.

Category structure. The category structure was the nonexhaustive two-category structure used by Saiki and Hummel (1996). There were two main categories: Hoz and Jik. They were nonexhaustive in that a nonmember of one category was not necessarily a member of the other. Thus, there was functionally a third category: Neither (i.e., neither Hoz nor Jik). Categories were defined by two three-valued substitutive attributes. Table 1 shows the relationships between the attributes and categories used in this experiment. In Table $1, h$ denotes an attribute value typical of $\mathrm{Hoz}, j \mathrm{de}-$ notes a value typical of Jik, and $n$ is typical of Neither (here, "attribute" refers to either part shape or relative location). The two relevant attributes were the shape of the variable part and its location relative to the common part. The three other attributes-the location of the object as a whole (randomized), the color of the common part (yellow), and the color of the variable part (red, blue, or green)-were irrelevant to category membership. There were nine different exemplar types, defined by the nine possible combinations of relevant attributes. Figure 2 shows examples of the exemplar types in the connected set. (Note that Figure 2 depicts only three instances per category -i.e., one instance of each exemplar type. In the experiments, there were 27 instances per category [nine instances of each of three exemplar types] because the variable part's color and the location of the object as a whole varied.) The color of the variable part was uncorrelated with category membership in that all nine exemplars were equally likely to have one of the three colors. Each row of Table 1 describes each exemplar type in terms of its frequency and attribute assignments. Typical members of the two main categories had typical values on both relevant attributes (exemplar type $h h$ for $\mathrm{Hoz}$ and $j j$ for Jik; Table 1). For example, a typical Hoz for one participant might have a triangle on top of the common part, and a typical Jik might have a circle left of the common part. For this participant, shape $h$ (the typical shape for Hoz) is triangle, relation $h$ is above, shape $j$ is circle, and relation $j$ is lefi-of. For a different participant, relation $h$ might 


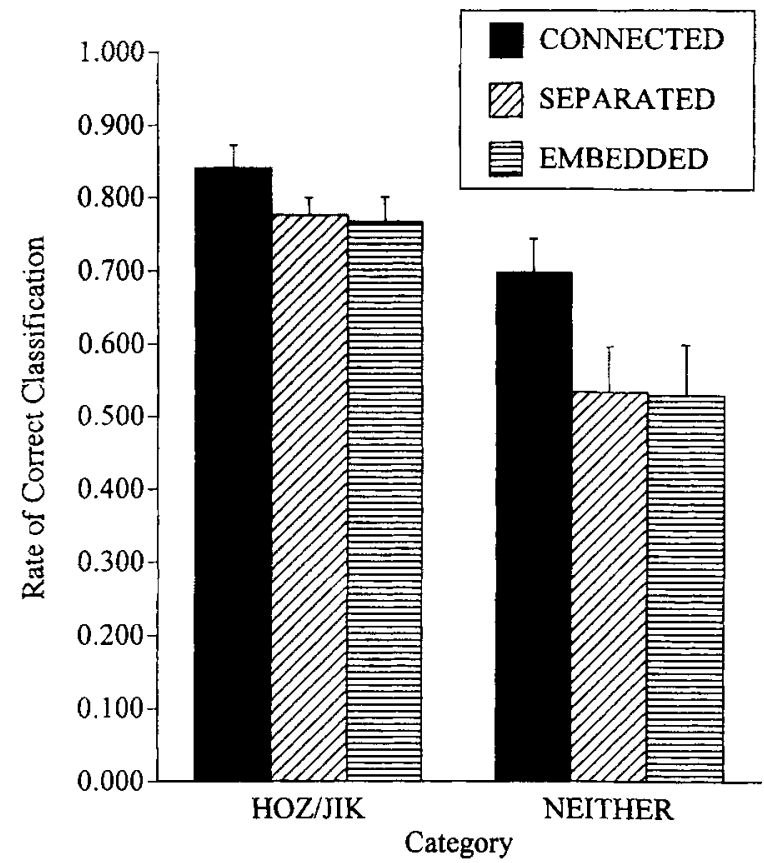

Figure 3. Mean correct classification rates for the three categories (Hoz and Jik are collapsed) in the connected, separated, and embedded conditions of Experiment 1. Error bars denote standard errors.

be right-of, and relation $j$ might be above. Objects in the Neither category were composed of either two attributes typical of neither Hoz nor Jik (shape $n$ and relation $n$; exemplar $n n$ in Table 1) or one attribute typical of Hoz and one typical of Jik (exemplars $h j$ and $j h$ in Table 1). Since the shape of the variable part was common to all stimulus sets, and since the category labels and shapes were completely arbitrary, the values of $h, j$, and $n$ were fixed for shapes (i.e., for all participants, $h=$ triangle, $j=$ circle, and $n=$ square). However, because the exact form of a relation varied across the stimulus sets (e.g., above in the connected condition is similar but not identical to above in the separated condition), typical values for relations were counterbalanced across participants. The logical category structure was fixed for all participants.

To make the task more difficult, we also included atypical members in the two main categories. These had one attribute typical of the main category ( $h$ for Hoz, and $j$ for Jik) and one attribute typical of the Neither category. Exemplars $h n$ and $n h$ were atypical members of $\mathrm{Hoz}$, and exemplars $j n$ and $n j$ were atypical members of Jik (Table 1). Twenty-two percent of the objects in each category were atypical. Consider a participant for whom the typical Hoz is a triangle (shape $h$ ) above (relation $h$ ) the rectangle, and a typical Jik is a circle (shape $j$ ) left of (relation $j$ ) the rectangle. For this participant, the atypical attributes are square (shape $n$ ) and top-right (relation $n$ ). Objects with a triangle on the right (type $h n$ ) and those with a square above (type $n h$ ) are atypical members of $\mathrm{Hoz}$, and objects with a circle on the right (type $j n$ ) and those with a square on the left (type $n j$ ) are atypical members of Jik. Objects with a square to the right (type $n n$ ), a triangle to the left (type $h j$ ), or a circle above (type $j h$ ) are members of Neither. Recall that performance on the Neither category indicates whether a participant is sensitive to both part shape and relative location: A participant can classify most members of Hoz and Jik correctly on the basis of part shape or relative location alone, but this strategy will cause them to systematically misclassify Neither objects. Therefore, per- formance on the Neither category is the critical dependent variable.

Procedure. The participants were tested individually. The experiment was run on a Macintosh IIC $i$ by a program written in MacProbe (Hunt, 1993). Trials were run in seven blocks of 27. Nine instances of each of the three categories were presented on each block. Irrelevant attributes were randomized, with the constraint that all values of each attribute appeared equally often (nine times per block). Presentation order was randomized. The participants were told that, on each trial, they would see an object and that their task was to predict the category to which it belonged. The instructions were the same for every participant, regardless of condition (connected, separated, and embedded), and emphasized that only one object would appear per trial. The participants were instructed to be as accurate as possible and to take as long as they needed to classify each object. They indicated that an object belonged to Hoz by pressing "H" on the keyboard, Jik by pressing "J," and Neither by pressing "N." Responses were followed by correct feedback. The drawing remained on the screen throughout the trial and during the feedback, so the participants could view the drawing and its category label as long as they wished. The participants initiated the next trial with a keypress. The next trial began after a $1-$ sec interval, during which the screen was blank. After each block, the computer showed the participants their percentage of correct responses, both for that block and for the experiment as a whole to that point.

\section{Results}

The data were analyzed in terms of the following measures: the overall rate of correct classification, the signal detection measure of discrimination $\left(d^{\prime}\right)$ between Neither category and the others, and the bias toward the common attribute (part shape or spatial relation). Throughout this paper, the overall rates of correct classification for $\mathrm{Hoz}$ and Jik were collapsed because they were very similar and because separating them was not informative.

Overall correct classification rates. Figure 3 shows the mean rates of correct classification for the categories in the three stimulus conditions. Since our theoretical interest was not to have an omnibus test but rather to compare the connected set with the separated and embedded sets, the statistical analyses were performed separately. Correct classification rates were lower in the separated condition than in the connected condition, especially for the Neither category. A 2 (stimulus set: connected vs. separated, between participants) $\times 2$ (category: Neither vs. $\mathrm{Hoz} / \mathrm{Jik}$, within participants) mixed-design analysis of variance (ANOVA) revealed a significant main effect of stimulus set $\left[F(1,22)=4.68, M S_{\mathrm{e}}=0.03, p<.05\right]$, a significant main effect of category $\left[F(1,22)=52.72, M S_{\text {e }}\right.$ $=0.01, p<.0001]$, and a marginally significant stimulus set $\times$ category interaction $\left[F(1,22)=3.46, M S_{\mathrm{e}}=0.01\right.$, $p=.076]$. Because the interaction was marginally significant, we tested the simple main effect of stimulus set for each category. For the Neither category, the rate of correct classification was significantly higher in the connected condition than in the separated condition $[F(1,22)$ $\left.=4.84, M S_{\mathrm{e}}=0.03, p<.05\right]$. However, for Hoz/Jik, the simple main effect failed to reach significance $[F(1,22)=$ $\left.2.92, M S_{\mathrm{e}}=0.01, p>.1\right]$. The participants in the sepa- 


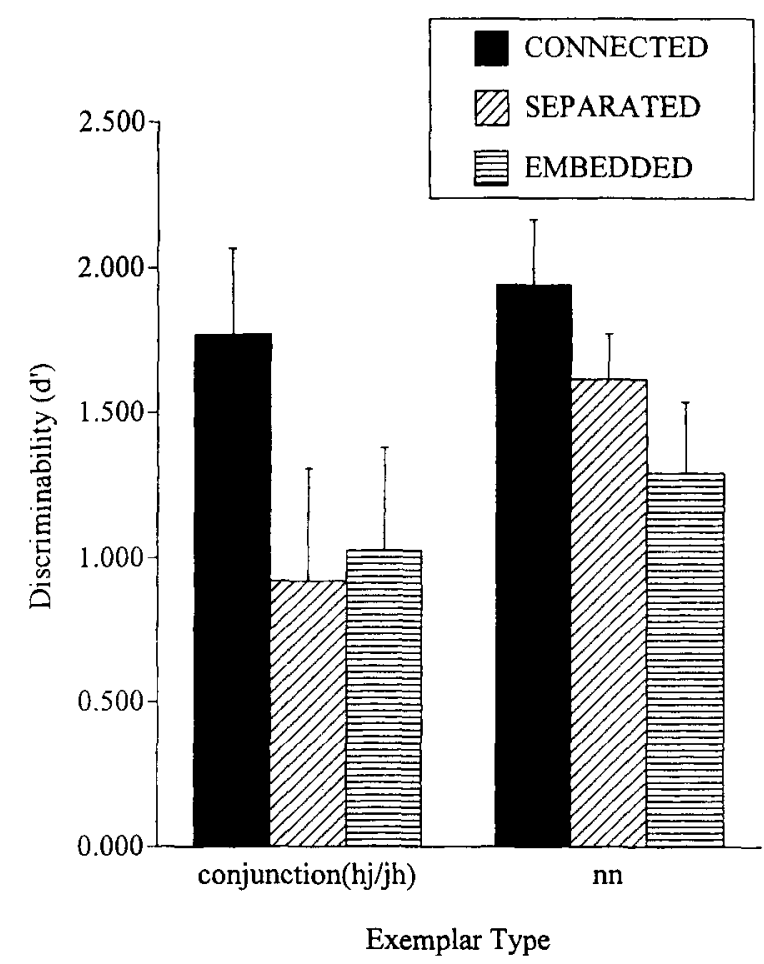

Figure 4. Mean $d^{\prime}\left(\right.$ conjunction) and $d^{\prime}(n n)$ in the connected, separated, and embedded conditions of Experiment 1. Error bars denote standard errors.

rated condition were significantly less accurate classifying the objects than were the participants in the connected condition, especially with the Neither category. This pattern suggests that the participants in the connected condition were better able to categorize objects on the basis of part-relation conjunctions than were the participants in the separated condition.

The results in the embedded condition were similar to those in the separated condition. The overall rate of correct classification was lower in the embedded condition than in the connected condition. A 2 (stimulus set: connected vs. embedded) $\times 2$ (category) mixed-design ANOVA revealed a significant main effect of stimulus set $[F(1,22)=$ 4.68, $\left.M S_{\mathrm{e}}=0.04, p<.05\right]$ and a significant main effect of category $\left[F(1,22)=43.97, M S_{\mathrm{e}}=0.01, p<.0001\right]$. The interaction was not statistically significant $[F(1,22)=$ $2.67]$. For the critical category - the Neither categorya single-factor ANOVA revealed a significant main effect of stimulus set $\left[F(1,22)=4.44, M S_{\mathrm{e}}=0.04, p<.05\right]$. Like the participants in the separated condition, the participants in the embedded condition had difficulty learning to categorize the objects on the basis of their part-relation conjunctions.

Detailed classification data. To further evaluate whether the differences between the connected and nonconnected (separated and embedded) conditions reflect the participants' use of part-relation conjunctions, we computed $d^{\prime}$ as a measure of the participants' ability to dis- criminate the members of the Neither category from those of Hoz and Jik: $d^{\prime}$ (conjunction) is an index of the participants' ability to discriminate $h j$ and $j h$ from members of Hoz and Jik, and $d^{\prime}(n n)$ is an index of the participants' ability to discriminate $n n$ from Hoz and Jik. The $d^{\prime}$ (conjunction) was the most critical measure for evaluating the participants' use of part-relation conjunctions. Figure 4 shows the mean $d^{\prime}$ 's for the three stimulus sets. As before, we performed the two comparisons (connected vs. separated, and connected vs. embedded) separately. Because $d^{\prime}$ increases rapidly at higher values of discriminability, we transformed the $d^{\prime}$ values by taking the natural logarithm of $\left(d^{\prime}+1.2\right)$. The log-transformed $d^{\prime}$ (conjunction) was significantly higher in the connected condition than in the separated condition $\left[F(1,22)=5.94, M S_{\mathrm{e}}=0.24\right.$, $p<.025]$. However, there were no significant differences with the log-transformed $d^{\prime}(n n)[F(1,22)=1.38$, $\left.M S_{\mathrm{e}}=0.04, p>.1\right]$, although performance in the connected condition was higher for both. The log-transformed $d^{\prime}$ (conjunction) was also significantly higher in the connected condition than in the embedded condition $\left[F(1,22)=4.91, M S_{\mathrm{e}}=0.18, p<.05\right]$. The log-transformed $d^{\prime}(n n)$ also showed significant differences $\left[F(1,22)=4.40, M S_{\mathrm{e}}=0.10, p<.05\right]$. Because $d^{\prime}$ (conjunction) is independent of the participants' bias to respond "Neither," these results suggest that the participants in the connected condition were better able to detect that $h j$ and $j h$ were not members of Hoz or Jik than were the participants in the separated and embedded conditions.

To investigate whether the differences among the conditions in $d^{\prime}$ (conjunction) and $d^{\prime}(n n)$ reflect the differences in earlier or later blocks, we calculated $d^{\prime}$ 's for earlier experimental blocks (Blocks 1-4) and for later experimental blocks (Blocks 5-7) separately. Mean hit rates, falsealarm rates, and estimated $d$ 's are shown in Table 2 . The differences between the conditions in $d^{\prime}$ were larger in the later blocks than in the earlier blocks, suggesting that the effect of connectedness does not simply reflect a bias to think of nonconnected stimuli as pairs of objects.

Analyses of errors. To assess whether the participants' errors reflected a bias to categorize on the basis of part shape only, relative location only, or neither, we estimated stimulus discriminability based on part shape and based on spatial relations. The measure of discriminability for part shape [ $d^{\prime}$ (shape)] was estimated as follows. "Hoz" responses to $h h$ and "Jik" responses to $j j$ were scored as hits, and responses consistent with an exemplar's relation but not its part shape (i.e., "Jik" responses to $h j$ and "Hoz" responses to $j h$ ) were scored as false alarms. This measure gives high false alarms, and therefore low $d^{\prime}$ 's, when a participant is biased against using shape as the basis for classification. The measure of discriminability for relative location $\left[d^{\prime}\right.$ (relation)] was estimated by using the same hit responses, but by using incorrect responses based on part shape (i.e., "Hoz" to $h j$ and "Jik" to $j h$ ) as false alarms. The participants who were biased to respond on the basis of part shape would have higher $d^{\prime}$ (shape) 
Table 2

\begin{tabular}{|c|c|c|c|c|c|c|c|c|c|c|}
\hline \multirow[b]{3}{*}{ Condition } & \multicolumn{10}{|c|}{$\begin{array}{c}\text { Mean Hit(conjunction), Hit(nn), False Alarm(Neither), } \\
d^{\prime} \text { (conjunction), and } d^{\prime}(n n) \text { for Early Blocks (Blocks 1-4) } \\
\text { and Late Blocks (Blocks 5-7) in Each Condition of Experiment } 1\end{array}$} \\
\hline & \multicolumn{2}{|c|}{$\operatorname{Hit}($ conj) } & \multicolumn{2}{|c|}{$\operatorname{Hit}(n n)$} & \multicolumn{2}{|c|}{ FA(Neither) } & \multicolumn{2}{|c|}{$d^{\prime}$ (conj) } & \multicolumn{2}{|c|}{$d^{\prime}(n n)$} \\
\hline & Early & Late & Early & Late & Early & Late & Early & Late & Early & Late \\
\hline \multicolumn{11}{|l|}{ Connected } \\
\hline$M$ & .53 & .88 & .67 & .81 & .16 & .07 & 1.14 & 3.06 & 1.54 & 2.83 \\
\hline$S D$ & .08 & .05 & .06 & .05 & .02 & .02 & 0.30 & 0.31 & 0.22 & 0.37 \\
\hline \multicolumn{11}{|l|}{ Separated } \\
\hline$M$ & .41 & .50 & .65 & .77 & .19 & .11 & 0.65 & 1.41 & 1.31 & 2.33 \\
\hline$S D$ & .09 & .12 & .05 & .08 & .02 & .02 & 0.33 & 0.60 & 0.16 & 0.37 \\
\hline \multicolumn{11}{|l|}{ Embedded } \\
\hline$M$ & .42 & .59 & .55 & .67 & .21 & .12 & 0.66 & 1.67 & 0.99 & 1.93 \\
\hline$S D$ & .08 & .09 & .06 & .08 & .02 & .02 & 0.32 & 0.46 & 0.23 & 0.41 \\
\hline
\end{tabular}

than $d^{\prime}$ (relation), and those biased to respond on the basis of relative location would have higher $d^{\prime}$ (relation) than $d^{\prime}$ (shape). The unbiased participants would have approximately equal $d^{\prime}$ (shape) and $d^{\prime}$ (relation). The mean $d^{\prime}$ (shape) and $d^{\prime}$ (relation) across conditions are shown in Figure 5, and mean $d$ 's, hit rates, and false-alarm rates in each condition are shown in Table 3 . The pattern of responses was quite different between the connected condition and the other two conditions. In the separated and embedded conditions, $d^{\prime}$ (relation) was higher than $d^{\prime}$ (shape), suggesting a bias to respond on the basis of relative location. The log-transformed $d^{\prime}$ (relation) was significantly higher than the log-transformed $d^{\prime}$ (shape) in these conditions [for the separated condition, $F(1,11)$ $=5.85, M S_{\mathrm{e}}=0.24, p<.05$; for the embedded condition, $\left.F(1,11)=7.21, M S_{\mathrm{e}}=0.29, p<.025\right]$. By contrast, in the connected condition, $d^{\prime}$ (shape) was slightly higher than $d^{\prime}$ (relation), and the difference was not statistically significant $\left[F(1,11)=0.39, M S_{\mathrm{e}}=0.06, p>.1\right]$.

\section{Discussion}

The participants' ability to correctly classify Neither exemplars is the best index of their ability to categorize on the basis of part-relation conjunctions (i.e., to integrate an object's parts with their relations for category learning). The results of Experiment 1 suggest that connectedness plays an important role in part-relation integration in object category learning. The participants in the connected condition were significantly better at classifying Neither exemplars than were the participants in the separated and embedded conditions. Performance was virtually the same in the embedded and separated conditions, suggesting that the superior performance in the connected condition does not simply reflect an effect of spatial proximity. Rather, it likely reflects the role of connectedness per se. Moreover, the poor classification performance in the embedded condition is incompatible with the hypothesis that the effect of connectedness is simply an effect of the perceived number of objects. To investigate this hypothesis more systematically, we asked 6 additional participants to rate the naturalness of the stimuli as single objects. All of them rated the embedded stimuli as the most natural, followed by the connected and separated stimuli. These ratings further suggest that the number of perceived objects cannot explain the results of Experiment 1 . The pattern of response biases reveals that the results cannot be attributed to a reduction in the salience of relations in the separated and embedded conditions: The participants in these conditions were more likely to classify objects on the basis of relative location alone than on the basis of part shape alone. These results are quite surprising given that the participants were allowed to view the objects for as long as they wished before making their category decisions.

The differences across conditions in the participants' ability to classify the Neither exemplars were especially pronounced in the later blocks. This result is important be-

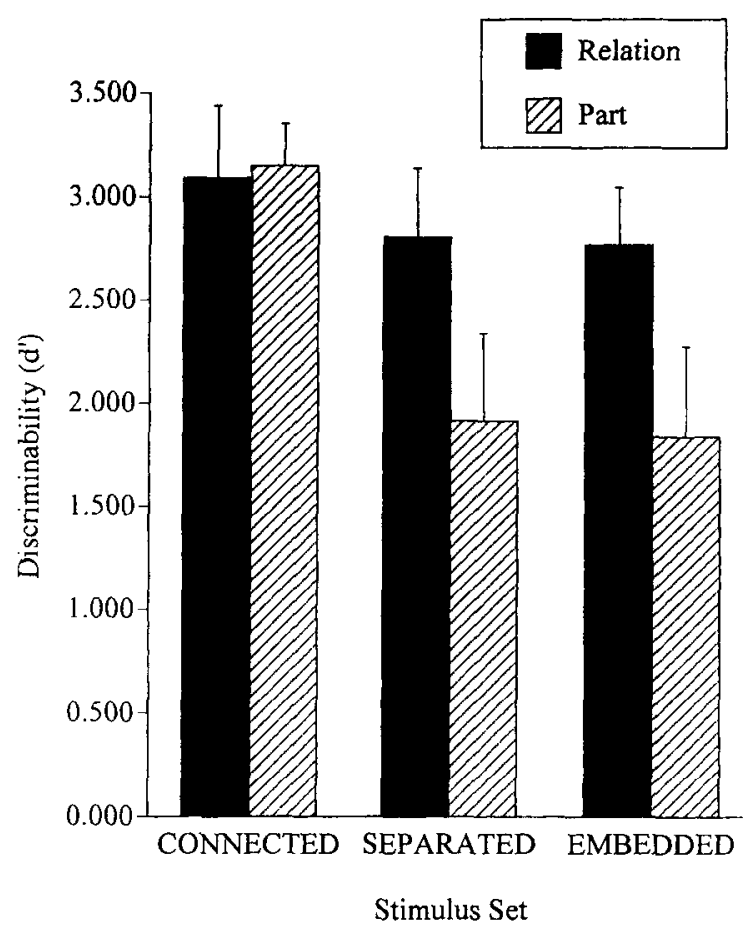

Figure 5. Mean $d^{\prime}$ (shape) and $d^{\prime}$ (relation) in the connected, separated, and embedded conditions of Experiment 1. Error bars denote standard errors. 
Table 3

Mean Hit, False Alarm(relation), False Alarm(shape), $d^{\prime}$ (relation), and $d^{\prime}$ (shape) in Each Condition of Experiment 1

\begin{tabular}{lccccc}
\hline Condition & Hit & FA(relation) & FA(shape) & $d^{\prime}$ (relation) & $d^{\prime}$ (shape) \\
\hline $\begin{array}{l}\text { Connected } \\
\quad\end{array}$ & & & & & \\
$M$ & .936 & .184 & .136 & 2.808 & 2.888 \\
$S D$ & .016 & .052 & .030 & 0.342 & 0.214 \\
Separated & & & & & \\
$M$ & .867 & .152 & .397 & 2.550 & 1.677 \\
$S D$ & .034 & .047 & .094 & 0.345 & 0.426 \\
Embedded & & & & & \\
$M$ & .839 & .118 & .390 & 2.506 & 1.574 \\
$S D$ & .039 & .026 & .075 & 0.302 & 0.431 \\
\hline
\end{tabular}

cause it suggests that the effect of connectedness is due, not to differences in the participants' initial strategies, but to differences in their evolving long-term memory representations of the stimulus categories.

There are some limitations of the stimuli used in Experiment 1 . First, because of the way the embedded and separated stimuli were generated from the corresponding connected stimuli, the possible locations of a variable part (e.g., on-top, left-of, etc.) were farther apart in the connected and separated conditions than in the embedded condition. Perhaps this made part-relation conjunctions less salient in the embedded condition than in the separated and connected conditions. A related problem is that the variable parts were larger in the connected and embedded conditions than they were in the separated condition. If large shapes are more discriminable than small shapes, then this may have favored performance in the connected and embedded conditions. Only the connected condition is favored by both of these potential confounds.

Experiment 2 replicated Experiment 1 with a stimulus set not subject to these criticisms. Experiment 2 also used verbal reports to gauge the conscious strategies that participants adopted in the connected, embedded, and separated conditions. If a naive coder can reliably sort participants into groups based on their conscious reports, then this would suggest that differences in performance across conditions may reflect the use of conscious strategies.

Another reason to replicate Experiment 1 concerns a "holistic processing" interpretation of the observed effects. Perhaps the participants learn better with connected stimuli than with separated or embedded stimuli because the former, but not the latter, can be processed "holistically" (e.g., because the parts and relations are perceptually more integral in the connected condition than in the other two conditions). By "holistic processing," we mean processing of the type that is performed on integral dimensions (Garner, 1974): an inability to respond to one stimulus dimension independently of another. For example, the brightness and the hue of a color are considered highly integral because it is very difficult to respond on the basis of brightness independent of hue, and vice versa. Equivalently, one could say that brightness and hue are processed holistically. Holistic representations differ from structured representations (such as a structural description) in that structured representations represent various dimensions--including relations and their arguments - independently (see Fodor \& Pylyshyn, 1988; Hummel \& Biederman, 1992; Hummel \& Holyoak, 1997). (As a result, structured representations, but not holistic ones, are compositional-forming complex structures by combining simple elements - and thus meaningfully symbolic.) If the stimuli in the connected condition are processed more holistically than are those in the separated and embedded conditions, then the task in the connected condition would be formally simpler than the task in the nonconnected conditions: The former would only require the participant to respond to a single holistic dimension (shape), whereas the latter would require the participant to integrate two separate dimensions (part shape and relative location). Although this account is not particularly plausible a priori, neither do the results of Experiment 1 strictly rule it out.

Experiment 3 used a variety of speeded classification tasks (specifically, those traditional in the literature of perceptual integrality) to test this account of the connectedness effect. If the perceptual integrality (i.e., "holisticness") of parts and relations differs across connected, separated, and embedded stimuli, then these differences should manifest themselves in traditional measures of perceptual integrality.

\section{EXPERIMENT 2}

The main purpose of Experiment 2 was to replicate Experiment 1 with a different set of stimuli. In Experiment 2, the positions and sizes of the variable parts were identical across the three stimulus sets. We manipulated the topological relation (connectedness, separatedness, and embeddedness) by manipulating the size of the common part. The common part was largest in the embedded set (i.e., large enough to fully surround all variable parts), smallest in the separated set (i.e., too small to touch any variable part), and medium-sized in the connected set (i.e., just large enough to touch each variable part). For any given participant, the common part and its topological relation to the variable part were again invariant. At the end of the experiment, we explicitly asked the participants about the strategies they used to perform the task. If the connectedness effect is due to the participants' use of conscious strategies, then the participants in the connected condition should report different strategies than the participants in the embedded and separated conditions. In particular, we examined whether the participants evidenced differential use of holistic information across conditions.

\section{Method}

Participants. Fifty-four undergraduates at UCLA participated for credit in introductory psychology classes. All participants had normal color vision. They were randomly assigned to experimental conditions.

Stimuli. Figure 6 shows the stimuli used in this experiment. The stimuli were similar to those of Experiment 1 , but there were sev- 
A. Connected Stimuli
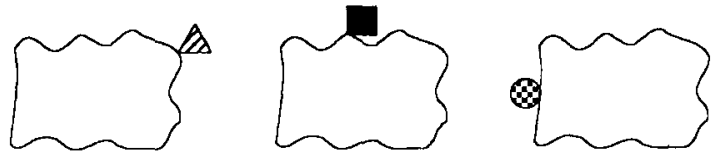

B. Separated Stimuli

$\Delta$<smiles>C1CCCCCCCCCCC1</smiles>



Q

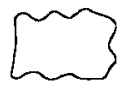

C. Embedded Stimul
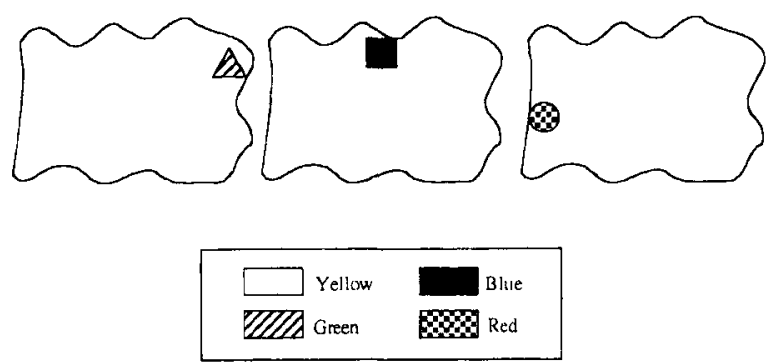

Figure 6. Examples of the stimuli used in Experiments 2 and 3.

eral differences. First, the overall size of the stimuli was substantially reduced in this experiment. The stimuli in the connected set occupied $25 \%$ of the area of the connected stimuli in Experiment 1. The common part of the connected set was $4.0 \mathrm{~cm}$ high and $6.0 \mathrm{~cm}$ wide, and the variable parts were approximately $1 \mathrm{~cm}$ high and $1 \mathrm{~cm}$ wide. The participants sat approximately $140 \mathrm{~cm}$ from the display, so the common and variable parts in the connected set subtended $1.8^{\circ} \times 2.7^{\circ}$ and $0.45^{\circ} \times 0.45^{\circ}$ of visual angle, respectively. Except for the objects' overall sizes, the connected set was almost identical to the connected set in Experiment 1 (Figure 6A). The exact locations of the variable parts (relative to the common part) in this experiment differed slightly from those of Experiment 1 because some adjustment was necessary to ensure that the variable parts touched the common part in both the connected set (where the variable touched the outside of the common part) and the embedded set (where the variable part touched the inside of the common part). The embedded set was created by enlarging the common part so that all variable parts were embedded within it, touching the interior of its bounding contour (Figure 6B). The common part of the embedded set was $47 \%$ larger than the common part of the connected set in both height and width. The separated set was created from the connected set by making the common part smaller. The common part was first reduced to $60 \%$ of its height and width in the connected set. It was then moved so that the distance between the common and variable parts was the same $(12 \mathrm{~mm})$ for all separated stimuli. In contrast to the variable parts in Experiment 1, all properties of the variable parts (i.e., their sizes, distances, and even exact locations on the computer screen) were identical across stimulus sets. The only thing that differed between the connected, separated, and embedded stimulus sets was the size of the common part and, as a result, its connectedness relations to the variable parts. The category structure was identical to that of Experiment 1. Un- like in Experiment 1, the typical values (i.e., $h, j$, and $n$ ) of both part shape and relative location were counterbalanced across participants.

Procedure. The procedure was identical to that of Experiment 1 , except that the participants answered an open-ended question about their use of strategies at the end of the experiment. The participants were asked to briefly explain how they learned the object categories and to describe any conscious strategies they used.

\section{Results}

Overall correct classification rate. The results of Experiment 2 replicated the basic results of Experiment 1: Performance was better in the connected condition than in the other two conditions. Figure 7 shows the mean rates of correct classification for the three categories in the three conditions. Statistical analyses were performed separately, as in Experiment 1. The rates of correct classification were higher in the connected condition than in the separated condition, especially for the Neither category. A 2 (stimulus set: connected vs. separated, between participants) $\times 2$ (category, within participants) mixeddesign ANOVA revealed a significant main effect of category $\left[F(1,34)=57.99, M S_{\mathrm{e}}=0.01, p<.0001\right]$, a marginally significant main effect of stimulus set $[F(1,34)=$ 4.03, $\left.M S_{\mathrm{e}}=0.03, p=.053\right]$, and a marginally significant interaction $\left[F(1,68)=3.93, M S_{\mathrm{e}}=0.01, p=.056\right]$. Because the interaction was marginally significant, we tested the simple main effect of stimulus set for each category. For the Neither category, the rate of correct classification was significantly higher in the connected condi-

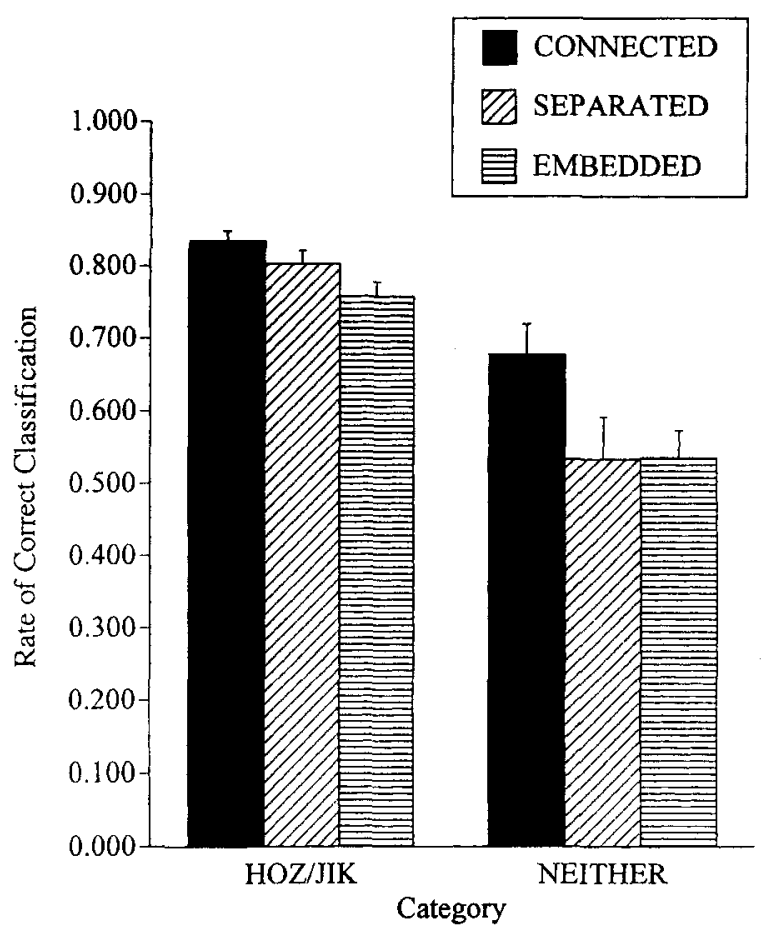

Figure 7. Mean correct classification rates for the three categories (Hoz and Jik are collapsed) in the connected, separated, and embedded conditions of Experiment 2. Error bars denote standard errors. 


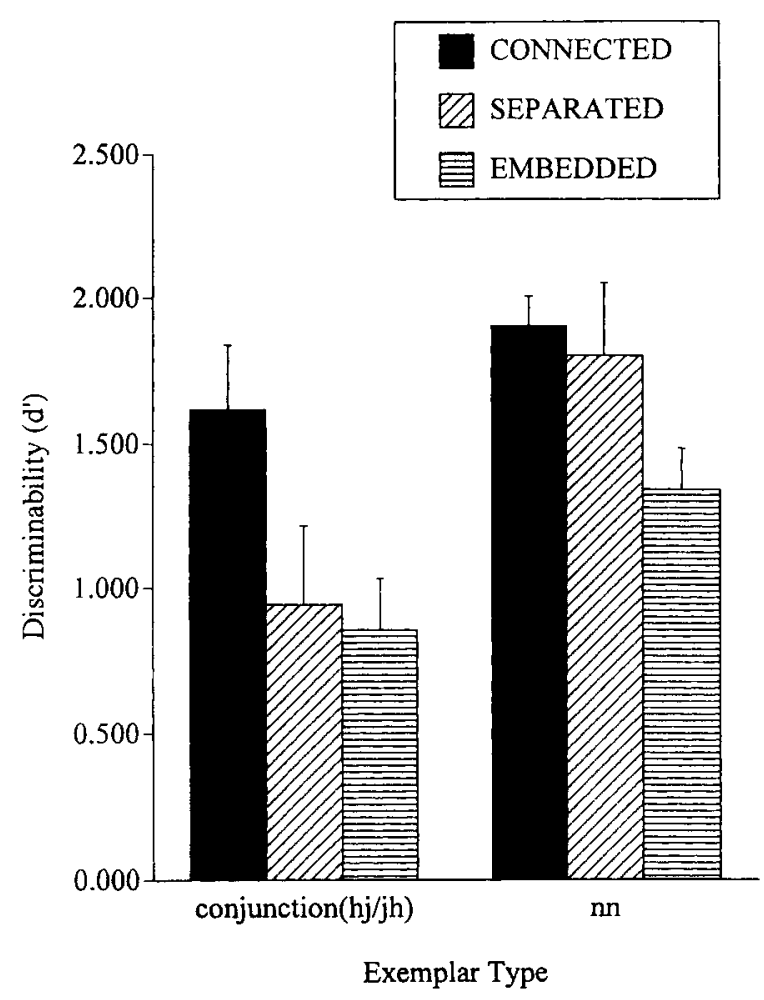

Figure 8. Mean $d^{\prime}$ (conjunction) and $d^{\prime}(n n)$ in the connected, separated, and embedded conditions of Experiment 2. Error bars denote standard errors.

tion than in the separated condition $\left[F(1,34)=4.32, M S_{\text {e }}\right.$ $=0.043, p<.05]$. The main effects of stimulus set were not significant for the other categories $[F(1,34)=1.64$, $\left.M S_{\mathrm{e}}=0.006\right]$. As in Experiment 1, the participants in the separated condition had more difficulty using part-relation conjunctions as a basis for categorization than did the participants in the connected condition.

Like performance in the separated condition, the rates of correct classification in the embedded condition were lower than those in the connected condition. A 2 (stimulus set) $\times 2$ (category) mixed-design ANOVA revealed a significant main effect of stimulus set $[F(1,34)=8.73$, $\left.M S_{\mathrm{e}}=0.02, p<.01\right]$ and a significant main effect of category $\left[F(1,34)=84.66, M S_{\mathrm{e}}=0.01, p<.0001\right]$. The interaction was not significant $[F(1,68)=2.31]$. For the critical category - the Neither category - a single-factor ANOVA revealed a significant main effect of stimulus set $\left[F(1,34)=6.58, M S_{\mathrm{e}}=0.027, p<.025\right]$. Like the participants in the separated condition, the participants in the embedded condition had difficulty categorizing objects on the basis of part-relation conjunctions.

Detailed classification data. Figure 8 shows the mean $d^{\prime}$ (conjunction) and $d^{\prime}(n n)$ for the three stimulus sets. Overall, the results were similar to those of Experiment 1. The statistically significant differences in the overall rate of correct classification for the Neither category were due mainly to exemplar types $h j$ and $j h$, indicating that the difficulty of the Neither category in the separated and embedded conditions reflects the difficulty of part-relation integration in those conditions. Table 4 shows the mean $d$ 's, hits, and false alarms for Blocks $1-4$ and 5-7. Unlike Experiment 1, the differences between the conditions obtained even in the earlier blocks; however, there were still significant effects of connectedness in the later blocks.

Analysis of errors. The mean $d^{\prime}$ (shape) and $d^{\prime}$ (relation) are shown in Figure 9 (see also Table 5). The pattern of biases is somewhat different from that of Experiment 1. First, only the separated condition revealed a strong bias toward relative location. Second, even the connected condition showed a weak (albeit nonsignificant) trend toward a relation bias. Planned comparisons between $d^{\prime}$ (shape) and $d^{\prime}$ (relation) in each condition revealed no significant difference in the connected condition $\left[F(1,17)=2.06, M S_{\mathrm{e}}\right.$ $=0.17, p>.1]$, suggesting neither part shape nor relation biases in the connected condition. In the separated condition, $d^{\prime}$ (relation) was significantly higher than $d^{\prime}$ (shape) $\left[F(1,17)=18.57, M S_{\mathrm{e}}=0.23, p<.0001\right]$, suggesting a relation bias. In contrast, in the embedded condition, $d^{\prime}$ (shape) and $d^{\prime}$ (relation) did not differ significantly $\left[F(1,17)=0.91, M S_{\mathrm{e}}=0.23, p>.1\right]$. Although all three conditions showed trends toward a relation bias (visible in Figure 9), this bias was statistically significant only in the separated condition.

Analysis of postexperiment questionnaire. All participants described the attributes that they used to categorize the objects. No participants stated that they learned categories completely by trial and error or that they sim-

Table 4

Mean Hit(conjunction), Hit(nn), False Alarm(Neither), $d^{\prime}$ (conjunction), and $d^{\prime}(n n)$ for Earty Blocks (Blocks 1-4) and Late Blocks (Blocks 5-7) in Each Condition of Experiment 2

\begin{tabular}{|c|c|c|c|c|c|c|c|c|c|c|}
\hline \multirow[b]{2}{*}{ Condition } & \multicolumn{2}{|c|}{ Hit(conj) } & \multicolumn{2}{|c|}{$\operatorname{Hit}(n n)$} & \multicolumn{2}{|c|}{ FA(Neither) } & \multicolumn{2}{|c|}{$d^{\prime}$ (conj) } & \multicolumn{2}{|c|}{$d^{\prime}(n n)$} \\
\hline & Early & Late & Early & Late & Early & Late & Early & Late & Early & Late \\
\hline \multicolumn{11}{|l|}{ Connected } \\
\hline$M$ & .56 & .74 & .66 & 88 & .17 & .07 & 1.18 & 2.54 & 1.43 & 3.02 \\
\hline$S D$ & .06 & .07 & .04 & .03 & .01 & .01 & 0.20 & 0.35 & 0.11 & 0.22 \\
\hline \multicolumn{11}{|l|}{ Separated } \\
\hline$M$ & .37 & .57 & .65 & .72 & .19 & .09 & 0.51 & 1.69 & 1.47 & 2.50 \\
\hline$S D$ & .07 & .09 & .05 & .08 & .02 & .02 & 0.25 & 0.40 & 0.22 & 0.40 \\
\hline \multicolumn{11}{|l|}{ Embedded } \\
\hline$M$ & .41 & .58 & .62 & .68 & .22 & .14 & 0.52 & 1.50 & 1.23 & 1.82 \\
\hline$S D$ & .04 & .07 & .05 & .05 & .02 & .02 & 0.15 & 0.31 & 0.22 & 0.25 \\
\hline
\end{tabular}


Table 5

Mean Hit, False Alarm(relation), False Alarm(shape), $d^{\prime}$ (relation), and $d^{\prime}$ (shape) in Each Condition of Experiment 2

\begin{tabular}{lccccc}
\hline Condition & Hit & FA(relation) & FA(shape) & $d^{\prime}$ (relation) & $d^{\prime}$ (shape) \\
\cline { 1 - 2 } $\begin{array}{c}\text { Connected } \\
\quad\end{array}$ & & & & & \\
$M$ & .929 & .123 & .241 & 2.989 & 2.533 \\
$S D$ & .011 & .041 & .061 & 0.205 & 0.286 \\
Separated & & & & & \\
$M$ & .883 & .094 & .451 & 2.909 & 1.525 \\
$\quad S D$ & .022 & .025 & .069 & 0.226 & 0.309 \\
Embedded & & & & & \\
$M$ & .855 & .211 & .308 & 2.113 & 1.742 \\
$S D$ & .020 & .044 & .054 & 0.223 & 0.224 \\
\hline
\end{tabular}

ply memorized all exemplars and their category labels. Rather, all participants' descriptions corresponded clearly to the attributes defined by the experimenters. Shape and color were described with the terms shape and color, respectively, and relative location was described with terms such as location, position, place, and so forth. No participants mentioned object features such as cusps (i.e., local concavities produced where the contours of separate parts meet), and no one mentioned overall shape or any similar term. The participants in all conditions described their strategies in terms of the attributes that we systematically varied, and there were no obvious differences between participants in different conditions in terms of how these attributes were described.

Table 6 shows the frequency with which the participants in each condition reported using various combinations of attributes. The patterns of attribute use are consistent with the classification data reported above. Of the participants who reported using part shape and relative location as the basis for classification, 11 were in the connected condition, 8 were in the separated condition, and only 5 were in the embedded condition.

To examine whether the participants' strategy reports reflected their category learning performance, we compared the overall rates of correct classification by the participants who reported using the correct combination of attributes (24 participants; henceforth called the correctreport group ) with the overall rates of correct classification by the participants who reported other combinations of attributes (30 participants; henceforth called the incorrectreport group). In the correct-report group, the mean rates of correct classification were $849(S D=.065)$ for the $\mathrm{Hoz}$ category, $.814(S D=.086)$ for the Jik category, and .707 $(S D=.160)$ for the Neither category. In the incorrectreport group, the mean rates of correct classification were $785(S D=.091)$ for the Hoz category, $.758(S D=.112)$ for the Jik category, and $.483(S D=.173)$ for the Neither category. In all three categories, the rates of correct classification were significantly higher for the correct-report group than for the incorrect-report group [for the Hoz category, $t(52)=2.89, p<.01$; for the Jik category, $t(52)$ $=2.02, p<.05$; for the Neither category, $t(52)=4.91$, $p<.001]$. Not surprisingly, the participants who reported using the correct attribute conjunctions for classification classified more objects correctly than did those who reported using incorrect combinations of attributes.

Interestingly, only one participant mentioned anything related to connectedness (such as separatedness, embeddedness, etc.) in his/her description of the stimuli: This participant used the word inside in his/her description of the stimuli. None of the participants' descriptions contained statements such as "I ignored the shape of the large part because it was separated from the small one," or "I paid attention to the features formed where the two parts came together." Apparently, none of the participants explicitly based their strategies on the connectedness or nonconnectedness of the stimuli they had to classify.

To further evaluate whether the participants' strategies differed between conditions, we asked a naive coder to judge which stimulus set each participant classified, solely on the basis of the participants' description of their strategies. The coder's task was to match each description to one stimulus set condition as accurately as possible, rating the confidence of his judgment. He was also instructed to report any aspect of each participant's description that explicitly suggested that the participant came from a particular stimulus set condition. The coder was given three sheets, each depicting the nine stimuli from one stimulus set in black and white. He was told about the basic design of the experiment, but not about the purpose of the experiment or the results. The basic logic behind

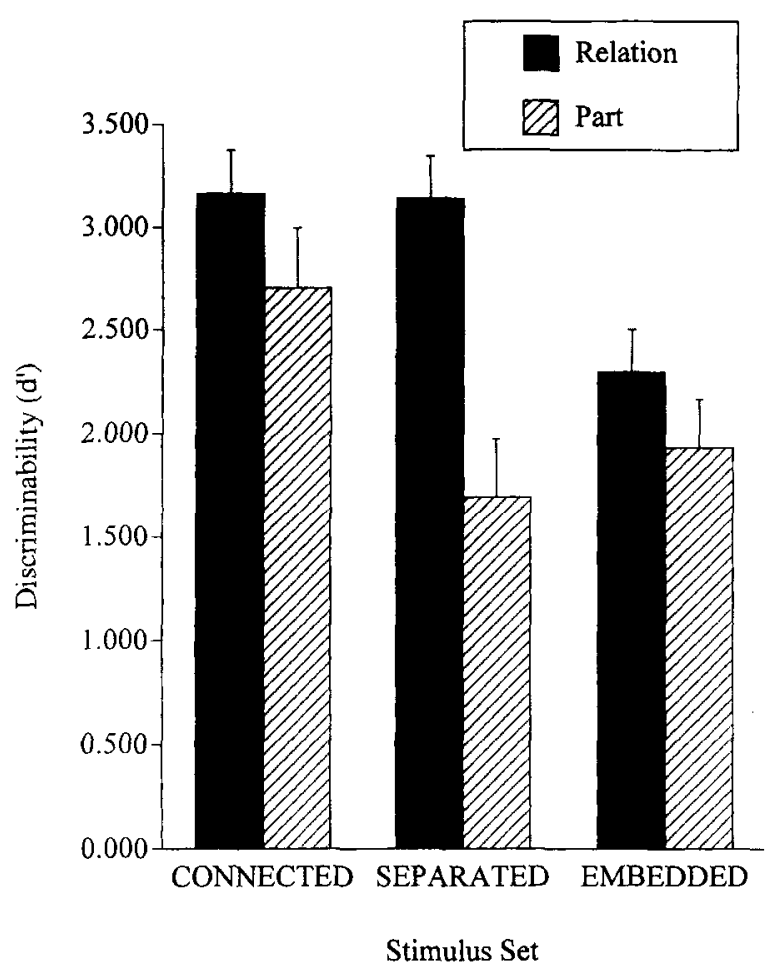

Figure 9. Mean $d^{\prime}$ (shape) and $d^{\prime}$ (relation) in the connected, separated, and embedded conditions of Experiment 2. Error bars denote standard errors. 
Table 6

Number of Participants in the Connected, Separated, and Embedded Conditions Who Reported Using Various Combinations of Attributes in Experiment 2

\begin{tabular}{lccc}
\hline & \multicolumn{3}{c}{ Condition } \\
\cline { 2 - 4 } \multicolumn{1}{c}{ Reported Attributes } & Connected & Separated & Embedded \\
\hline Location only & 3 & 4 & 2 \\
Shape only & 0 & 0 & 2 \\
Color only & 0 & 0 & 0 \\
Location and Color & 0 & 1 & 0 \\
Location and Shape* & 11 & 8 & 5 \\
Shape and Color & 2 & 0 & 2 \\
Location, Shape, and Color & 2 & 5 & 7 \\
Total & 18 & 18 & 18 \\
\hline
\end{tabular}

*The correct combination of attributes.

this coding task was the following. If the participants use conscious strategies related to connectedness, then these strategies might manifest themselves in the participants' descriptions, either explicitly or implicitly. If the descriptions contain information about the connectedness of the stimulus set, then the coder should be able to assign descriptions to stimulus set conditions with better-thanchance (or worse-than-chance) accuracy. Even if the coder cannot identify explicit evidence for the use of strategies related to connectedness, different-than-chance judgments could be interpreted as an indication of the participants' use of connectedness-related strategies.

The coder's mean accuracy was $31.5 \%$, which is not significantly different from chance $(33 \%)$. His rating performance reveals no evidence for the use of explicit strategies related to connectedness. The coder indicated explicit evidence for a participant's stimulus set condition in only 3 descriptions out of 54 . Two of these were drawings of the stimuli, and was the use of the word inside (noted previously), which suggests that the participant was in the embedded condition. However, the coder indicated that none of these pieces of "evidence" was related to the participant's description of his or her strategies.

\section{Discussion}

Experiment 2 replicated the connectedness effect of Experiment 1 . This replication discounts several alternative interpretations of the connectedness effect. First, the analysis of the participants' strategy reports suggests that the effect does not reflect the participants' use of explicit strategies related to connectedness. In particular, the fact that none of the participants mentioned the use of "overall shape" (or any similar term) suggests that the effect of connectedness does not reflect the conscious use of holistic information. However, the strategy report data should be interpreted with caution, because it is a weak test. Second, the effect was not due to characteristics of the stimuli, such as distances between variable parts or the sizes of the variable parts. Third, although the connectedness effect was small, it is unlikely to have been due to sampling error, because the main results replicated. The stimulus sets of Experiment 2 confounded connectedness with the size of the common part, but the fact that similar results were obtained in Experiments 1 and 2 suggests that the size of the common part (or the relative sizes of the common and variable parts) was unlikely to have been responsible for the connectedness effect. (Recall that the common part was a constant size across conditions in Experiment 1.) The fact that Experiments 1 and 2 gave similar results suggests that connectedness was the main factor responsible for the observed effects.

There were some differences between the results of Experiments 1 and 2, especially in the patterns of bias. The bias to categorize on the basis of relative location alone was weaker in the embedded condition of Experiment 2 than in the embedded condition of Experiment 1 . Although there is no clear explanation for this difference, one possibility is that it reflects the different embedded locations used in the experiments. In Experiment 1, the embedded set included one location in the center of the common part, with the other two locations touching the edge of the common part. In contrast, in Experiment 2, the variable part touched the edge of the common part in all three embedded locations. This qualitative difference in the population of embedded locations may account for the different pattern of biases across Experiments 1 and 2 . Alternatively, the difference may simply reflect sampling error. What is more important for our present purposes is the fact that neither experiment revealed any bias to respond on the basis of part shape. This is important because it suggests that the observed effects of stimulus set cannot be attributed to simple differences in the salience of relative location.

Although the participants' strategy reports suggest that the connectedness effect does not reflect differences in their conscious strategies, it remains possible that the connectedness effect reflects the use of holistic information at an unconscious level. Evidence of dimensional integrality has often been interpreted as evidence of holistic processing (Barrett \& Shepp, 1988; Kemler Nelson, 1993; Lockhead, 1966; Shepp \& Barrett, 1991). It is possible that part shape and relative location were more integral (and therefore processed more holistically) in our connected stimuli than in our separated and embedded stimuli. Another potential explanation of the connectedness effect is that it reflects subtle differences in the salience of part shape and relative location (as single attributes) across our connected and nonconnected stimuli: If the parts' shapes and/or relations are more salient in the connected stimuli than in the nonconnected stimuli, then this difference could make category learning easier in the former condition than in the latter. Experiment 3 used a speeded classification task to test the dimensional integrality and the single attribute salience accounts of the connectedness effect.

\section{EXPERIMENT 3}

Speeded classification is a standard paradigm for testing dimensional integrality (Garner, 1974). Following 
Pomerant $z$ and Pristach (1989), Experiment 3 used nine classification tasks to evaluate dimensional integrality: four control tasks, two selective attention (filtering) tasks, two correlated dimensions (redundancy) tasks, and one divided attention (condensation) task. In a filtering task, the participants must respond to one stimulus dimension, ignoring the other. Here, categories are defined by part shape alone or spatial relations alone, although both dimensions vary across stimuli. In the redundancy task, the dimensions are redundant in that the participant can respond correctly on the basis of one or the other alone. In the divided attention task, the participant must attend to both dimensions to respond correctly, but their values are crossed, making the categories nonlinearly separable (e.g., both shape-1/relation-1 and shape-2/relation-2 belong to Category A, but both shape-1/relation-2 and shape-2/relation-1 belong to Category B). There were four control tasks, described shortly.

To the degree that part shape and relative location are integral in a given stimulus set, that set should reveal three patterns of effects: (1) a filtering loss, in which responses are slower in the filtering task than in the control task, (2) a redundancy gain, in which responses are faster in the correlated task than in the control task, and (3) a divided attention loss, in which responses are slower in the divided attention task than in the control task. To the degree that part shape and relative location are separable in a stimulus set, that set should reveal no filtering loss, no redundancy gain, but a large divided attention loss. To the degree that part shape and relative location are configural in a stimulus set (Pomerantz \& Pristach, 1989), that set should reveal a filtering loss, no redundancy gain, and no divided attention loss. If our connected, separated, and embedded stimulus sets differ with regard to the integrality or configurality of part shape and relative location, then they will reveal different patterns of these effects. Such differences would suggest that the connectedness effect can be understood in terms of standard dimensional integrality or configurality.

Speeded classification is also a good way to evaluate the salience of part shape and location as single attributes. Response time is the main dependent variable in the speeded classification task. Salience differences that are too small to detect in unspeeded classification may nonetheless manifest themselves as different response times in a speeded classification task. If the connectedness advantage is due to small differences in the salience of relative location or part shape across conditions, then responses will be faster in the connected condition than in the embedded and separated conditions.

\section{Method}

Participants. Forty-eight undergraduates at UCLA participated for credit in introductory psychology classes. All had normal color vision.

Design. There was one between-participant factor, stimulus set, with four conditions: connected, separated, embedded, and shapecolor. The first three conditions used the corresponding stimuli from Experiment 2 and evaluated the dimensional integrality of part shape and relative location. The shape-color condition served as a baseline against which to evaluate separability, because shape and color are known to be separable dimensions. There was one within-subjects factor, classification task, with nine conditions as elaborated below.

Stimuli. The stimuli were identical to those of Experiment 2. The connected set was used in the shape-color condition.

Classification tasks. Figure 10 illustrates the dimensional structure and classification tasks used in this experiment. For each participant, categories were defined by two values of each attribute (e.g., red and green; circle and triangle; beside and on top). These values are denoted S1 and S2 for part shape, R1 and R2 for relative location, and $\mathrm{C} 1$ and $\mathrm{C} 2$ for color. The nine classification tasks were defined by the assignment of instances to categories. In the control tasks, categories were defined by the value of only one attribute, with the value of the other attribute fixed. There were four control tasks. The two shape control tasks had fixed values of relative location, either $\mathrm{R} 1$ (the shape-RI task) or R2 (the shape-R2 task). The two relation control tasks had fixed values of part shape, either S1 (the relation-S1 task) or S2 (the relation-S2 task). For example, in the shape-R1 task, S1R 1 belonged to Category $A$, and S2R1 belonged to Category $B$; in the shape-R2 task, S1R2 belonged to Category A, and S2R2 belonged to Category B. Categories in the correlated tasks also contained two instances, but here the instances were defined by both attributes. There were two correlated tasks: the $11 / 22$ correlated task, which was made up of S1R1 (Category A) and S2R2 (Category B); and the 12/21 correlated task, which was made up of S1R2 (Category A) and S2R1 (Category B). In both cases, categories were defined redundantly, making it possible to use either part shape or relative location (or both) as the basis for classification. Categories in the selective attention tasks (also called filtering tasks) were defined by one attribute, with the other attributes irrelevant. There were two filtering tasks: in the shape filtering task, S1R1 and S2RI belonged to Category A, and S1R2 and S2R2 belonged to Category B; in the relation filtering tasks, $\mathrm{S} 1 \mathrm{R} 1$ and $\mathrm{S} 1 \mathrm{R} 2$ belonged to Category $\mathrm{A}$, and S2R1 and S2R2 belonged to Category B. Categories in the divided attention task (also called a condensation task) were defined by a combination of values on both dimensions: S1R 1 and S2R 2 belonged to Category A, and S1R2 and S2R1 belonged to Category B. In the shape-color condition, the classification tasks were defined by replacing the relation attribute with the color attribute. Otherwise, the tasks were identical to those described above. Figure $10 \mathrm{~B}$ summarizes the assignment of objects to categories in each classification task.

From the stimulus set used in Experiment 2, which had three values on all the attributes, two values of each attribute were selected for each participant. The selected values were counterbalanced across participants.

Procedure. The participants were run individually on a Macintosh IIfx computer with a color monitor. The participants sat approximately $140 \mathrm{~cm}$ from the monitor, and they were asked to hold their heads in a constant position. The experiment room was dimly lit.

The experiment consisted of 27 blocks, each with 24 trials. At the beginning of each block, the participants were told about each object's category membership. The screen was divided by a vertical line, with members of Category A (either one or two, depending on the task) displayed on the left side and the members of Category B (either one or two) on the right. The participants were allowed to study the display for as long as they wished. The speeded classification trials were initiated by a keypress from the participant. The 24 trials in a block contained 12 Category A trials and 12 Category B trials. All instances appeared equaily often. The order of trials was randomized within blocks. One task was used on each block, with the nine tasks grouped into nine-block sets. The whole experimental session consisted of three repetitions of each nine-block set. The order of classification tasks was randomized within each set. 
A. Dimensional Structure and Exemplars

\begin{tabular}{|c|c|c|c|}
\hline & \multicolumn{2}{|c|}{ Relation } \\
\hline & & R1 & R2 \\
\hline \multirow{2}{*}{ Shape } & S1 & SIRI & S1R2 \\
\hline & $\mathrm{S} 2$ & $\mathrm{~S} 2 \mathrm{R} 1$ & S2R2 \\
\hline
\end{tabular}

B. Category Membership of Classification Tasks
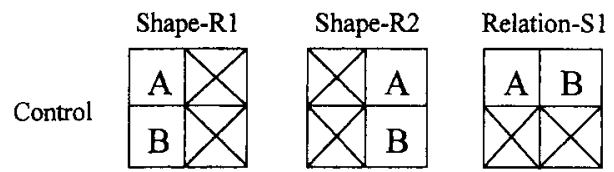

Relation-S2

$11 / 22$ correlated $12 / 21$ correlated
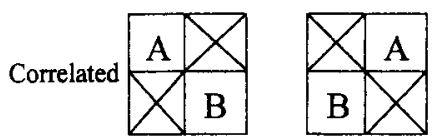

Shape filtering

Relation filtering

\begin{tabular}{l|l|l|} 
Selective & A & B \\
\hline Attention & A & B \\
\hline
\end{tabular}

\begin{tabular}{|c|c|}
\hline $\mathrm{A}$ & $\mathrm{A}$ \\
\hline $\mathrm{B}$ & $\mathrm{B}$ \\
\hline
\end{tabular}

Figure 10. Dimensional structure and category membership used in Experiment 3. Panel A shows how each exemplar type was defined; Panel B shows how exemplars were assigned to categories in each classification task. For example, exemplars S1R1 and S2R1 were assigned to Categories $A$ and $B$, respectively, in the shape-R1 task. In the shape-color condition, relation is replaced with color.

The first nine-block set was practice, and the results from that set are not included in the data analyses.

Each trial began with the presentation of an object in one of four quadrants on the screen (randomly chosen). The object remained on the screen until the participant responded by pressing a button on a button box (the left and right buttons were for Categories $A$ and $\mathrm{B}$, respectively). The participants were instructed to respond as quickly and accurately as possible. After the response, feedback was displayed for $500 \mathrm{msec}$. When the response was correct, the word right was presented along with the response time; incorrect responses were followed by the word wrong without a response time. The intertrial interval was approximately $500 \mathrm{msec}$. The participant's mean rate of correct classification and mean response time were displayed on the screen at the end of each block.

\section{Results}

The control, filtering, and correlation tasks all had very similar response times within experimental conditions. Figure 11 shows the collapsed means across these tasks, along with the mean response time for the divided attention task. The pattern of response times across tasks was virtually identical across the four stimulus sets: Neither the integrality nor the configurality of part shape and relative location varied as a function of connectedness (stimulus set). Moreover, part shape and relative location showed the same pattern as part shape and color. Because shape and color are known to be separable, this pattern suggests that part shape and relative location are also separable. Response times in the control tasks also did not vary as a function of stimulus set: The salience of part shape and relative location was unaffected by connectedness. Indeed, although the difference was not statistically reliable, response times were slowest in the connected condition, further suggesting that the connectedness effect cannot be explained in terms of even a small salience advantage in the connected condition relative to the separated and embedded conditions.

These patterns, visible in Figure 11, were supported by the statistical analyses. To evaluate dimensional integrality, we first ran a 4 (stimulus set) $\times 9$ (classification task) mixed-design ANOVA. Any significant differences between patterns of response times across tasks across stimulus sets would manifest themselves as an interaction between stimulus set and classification task. The ANOVA revealed significant main effects of stimulus set $[F(3,44)=$ $\left.4.04, M S_{\mathrm{e}}=28,192.15, p<.025\right]$ and task $[F(1,44)=$ $\left.269.14, M S_{\mathrm{e}}=1,806.88, p<.0001\right]$, but the interaction did not approach statistical significance $[F(3,44)=$ 1.18]. The main effect of stimulus set reflects the shorter response times in the shape-color condition. When the shape-color condition is excluded from the analysis, the main effect of stimulus set disappears $[F(2,33)=0.36]$. A separate set of ANOVAs with collapsed response times showed the same pattern of results, which revealed no significant interaction between stimulus set and task $(F \mathrm{~s}<1)$. The fact that stimulus set did not interact with task indicates that the stimulus sets do not differ in their dimensional integrality.

To further evaluate whether integrality differed across conditions, we calculated filtering interferences and redundancy gains, following Ashby and Maddox (1994). Interference due to filtering location (or color) was calculated by subtracting the mean response times in the shape- $\mathrm{R} 1$ (or shape- $\mathrm{C} 1$ ) and shape-R2 (or shape-C2) tasks from the mean response time in the relation (or color) filtering task. Interference due to filtering part shape was calculated by subtracting the mean response times in the relation-S1 (or color-S1) and relation-S2 (or color-S2) tasks from the mean response time in the shape filtering task. The resulting differences indicate the relation (or color) filtering loss and the shape filtering loss. Positive values indicate interference, and negative values indicate facilitation. Redundancy gains were calculated by subtracting the mean response times in either the $11 / 22$ correlated or the $12 / 21$ correlated tasks from the mean response times in the four control tasks. The resulting differences indicate, respectively, the $11 / 22$ redundancy gain and the 12/21 redundancy gain. Positive values indicate facilitation. We calculated the divided attention loss (i.e., cost in the divided attention task) by subtracting the mean response times in the four control tasks from the mean response time in the condensation task. Positive values indicate a divided attention loss. Mean gains and losses are shown in Table 7. 


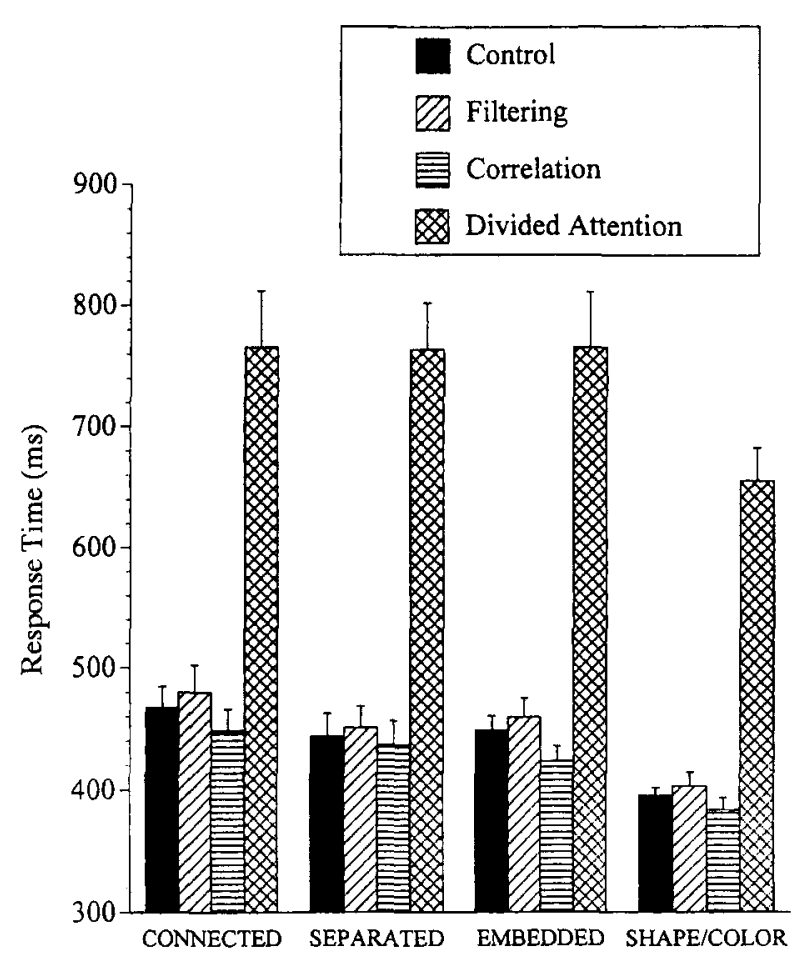

Stimulus Set

Figure 11. Mean response times for four classification tasks in the connected, separated, embedded, and shape-color conditions of Experiment 3. Error bars denote standard errors.

Following Experiments 1 and 2, the separated and embedded conditions were compared with the connected condition separately. Neither comparison of the connected and separated conditions nor comparison of the connected and embedded conditions revealed any significant differences on any loss or gain measures.

To test single-attribute salience across stimulus sets, we compared response times in the four control tasks across stimulus set. A 3 (stimulus set) $\times 4$ (task) mixed-design ANOVA revealed no significant main effects and no interaction $(F<1)$. Separate comparisons of the connected and embedded conditions and of the connected and separated conditions showed the same pattern (all $F \mathrm{~s}<1$ ).

\section{Discussion}

The results of Experiment 3 are clearly inconsistent with the hypothesis that the integrality of part shape and relative location differs between the connected, separated, and embedded stimulus sets. Rather, they suggest that part shape and relative location are separable in all three stimulus sets. Compared to typical integral dimensions, which show 50-100 msec of filtering loss (Felfoldy, 1974; Grau \& Kemler Nelson, 1988; Pomerantz \& Pristach, 1989 ), the filtering losses in all the conditions were very small (on the order of 10-15 msec). Kimchi and Palmer (1985), using hierarchically organized part-whole stimuli, also observed small filtering losses $(17-21 \mathrm{msec})$ and redundancy gains (8-24 msec) between part and whole shapes, and, in contrast to us, interpreted these patterns as evidence of integral dimensions. However, most of the losses and gains we observed were even smaller than those observed by Kimchi and Palmer. More importantly, the shape-color condition showed losses and gains similar in magnitude to those observed in the other conditions: Their comparable losses and gains suggest that part shape and relative location are as separable as shape and color (which are considered highly separable). Because integrality did not differ across stimulus sets, the results of Experiment 3 are inconsistent with the hypothesis that integrality is responsible for the connectedness effect, even on an extremely liberal criterion of integrality. Finally, the large condensation cost observed in all three stimulus conditions suggests that the connectedness advantage cannot be explained by appeal to the hypothesis that a part's shape and location are configural dimensions (Pomerantz \& Pristach, 1989).

The data are also inconsistent with the hypothesis that part shape, relative location, or both are more salient in the connected set than in the other sets. In fact, although the difference was not statistically significant, the mean response times were actually longer in the connected condition than in the other conditions. If salience differs at all across stimulus sets, then it is lower in the connected set.

Taken together, these findings show that the connectedness effect observed in Experiments 1 and 2 cannot be explained in terms of dimensional integrality, dimensional configurality, or small differences in salience.

Table 7

Filtering Loss, Redundancy Gain, and Divided Attention Loss (in Milliseconds) for Stimulus Set Conditions in Experiment 3

\begin{tabular}{|c|c|c|c|c|c|c|c|c|}
\hline \multirow[b]{3}{*}{ Exemplar Type } & \multicolumn{8}{|c|}{ Stimulus Set } \\
\hline & \multicolumn{2}{|c|}{ Connected } & \multicolumn{2}{|c|}{ Separated } & \multicolumn{2}{|c|}{ Embedded } & \multicolumn{2}{|c|}{ Shape-Color } \\
\hline & $M$ & $S D$ & $M$ & $S D$ & $M$ & $S D$ & $M$ & $S D$ \\
\hline Relation filtering loss & 11 & 25 & 1 & 36 & -6 & 27 & 7 & 28 \\
\hline Shape filtering loss & 14 & 35 & 14 & 30 & 27 & 23 & 8 & 23 \\
\hline $11 / 22$ redundancy gain & 19 & 33 & 7 & 38 & 25 & 18 & 14 & 20 \\
\hline $12 / 21$ redundancy gain & 18 & 26 & 7 & 26 & 26 & 12 & 10 & 27 \\
\hline Divided attention loss & 299 & 113 & 320 & 87 & 317 & 130 & 260 & 80 \\
\hline
\end{tabular}

Note-In the shape-color condition, the data in the row labeled "Relation filtering loss" indicate color filtering loss. 


\section{GENERAL DISCUSSION}

The present series of experiments showed that connectedness plays an important role in people's ability to learn categories defined by parts in particular spatial relations. In Experiment 1, the participants were tested for their ability to learn shape categories defined by partrelation conjunctions (the shape of one single part and its location relative to another part). Performance was better when the parts in the to-be-learned categories were connected than when they were separated or embedded. Rather than responding on the basis of part-relation conjunctions (the defining attributes of the categories), the participants in the separated and embedded conditions revealed a bias to respond on the basis of relative location alone (rather than part shape). This bias suggests that the effect of connectedness does not simply reflect the participants' ignoring relative location in the separated and embedded conditions. Experiment 2 replicated the main results of Experiment 1 and showed that the connectedness effect was not due to the participants' use of conscious strategies related to connectedness. No participant reported using overall shape as a basis for classification, suggesting that the connectedness effect is not related to the conscious use of holistic shape information. In Experiments 1 and 2, the connectedness advantage was more pronounced in later blocks than in earlier blocks. This effect suggests that the connectedness effect reflects the participants' memory representations of the object categories, rather than their initial (conscious or unconscious) strategies. Experiment 3 more systematically investigated the possible role of holistic processing in the connectedness effect by examining the dimensional integrality of part shape and relative location. This experiment showed that part shape and relative location are equally (and strongly) separable in the connected, separated, and embedded stimulus sets. Furthermore, the analysis of response times in the control tasks suggests that the connectedness effect cannot be attributed to the salience of parts and relations as single attributes. Rather, the connectedness effect manifests itself specifically in the participants' sensitivity to part-relation conjunctions in the formation of category representations (equivalence classes).

Taken together, these findings suggest that people are more likely to integrate an object's parts with their relations for categorization when they are connected than when they are separated or embedded. These findings are important because they show that connectedness affects part-relation integration in category learning, even with unlimited viewing time: Apparently, the role of perceptual grouping in the integration of different attributes extends beyond early perceptual binding. More broadly, these findings suggest that perceptual constraints on partrelation integration can affect the "higher" cognitive process of category learning: As noted by Barsalou (1993) and others (e.g., Humphreys \& Bruce, 1989), perceptual constraints deserve attention in theoretical accounts of categorization and category learning.

\section{What Is Connectedness?}

We have used the term connectedness to refer to a visual cue suggesting the physical connectedness of two parts. Although this is not a formal definition of connectedness, it is largely consistent with the findings of this work, and this conception of connectedness has some broader implications. First, connectedness is not identical to spatial proximity. Performance in the embedded condition (where the parts were very close to one another) was like performance in the separated condition and was unlike performance in the connected condition. A related point is that simply occupying overlapping regions in the image is not sufficient to obtain the facilitatory effect of connectedness: Classification performance differed substantially between the connected and embedded conditions in Experiments 1 and 2, even though the parts of a stimulus touched each other (in the image) in both cases. At the same time, however, all six independent raters described the embedded stimuli as single objects. Together, these facts suggest that the difficulty in the embedded condition may reflect the participants' interpreting the embedded parts as surface markings (e.g., analogous to the black spots of a Dalmatian), rather than as distinct parts. Perhaps the human object categorization system is less concerned with the spatial relations between an object's parts and their surface markings than with the relations between one part and another. Intuitively, this kind of bias seems sensible, since the relations between the parts are more informative about the properties of an object (such as whether it affords sitting) than are the locations of its surface markings (such as where flowers are embroidered on the seat). Further study is necessary to determine what factor is responsible for the difficulty of category learning in the embedded condition, but the difficulty of the embedded and separated conditions suggests two important conditions for connectedness to facilitate part-relation binding in object category learning: The elements must both be interpreted as parts, and they must be interpreted as physically connected in the 3-D world. The embedded objects seem to violate the first condition, and the separated objects violate the second.

\section{Holistic Information, Emergent Features, and Part-Relation Integration}

Although the results of Experiment 3 speak against a simple perceptual integrality account of the connectedness effect, we should note that there is a more sophisticated variant of the holistic account that our data are insufficient to falsify. Dimensional integrality, as measured by speeded classification tasks, reflects the mandatory use of holistic information. Thus, even if parts and relations are perceptually separable in this strong sense of "integral" (vs. separable), it remains possible that connected and nonconnected stimuli differ in a weaker form of part-relation integrality. For example, perhaps the participants in our connected conditions were somehow better able to use holistic information when it was informative. 
Although this type of modified holistic account may be correct, it requires cautious analysis. The term informative requires clarification. If holistic features are informative in the usual sense, then there should have been a redundancy gain with the connected stimuli of Experiment 3; if such informative features are absent in the separated or embedded stimuli, then there should have been a difference between the connected and nonconnected conditions with regard to the magnitude of this redundancy gain. Experiment 3 revealed neither of these effects. If we are to assume that the participants selectively use informative features, then it becomes important to explain why they used them in Experiments 1 and 2 but not in Experiment 3 . There might be some other (as yet unknown) reason for this difference, but, for now, it seems more parsimonious simply to assume that connectedness effect is not due to the availability of holistic information but rather reflects the processes whereby we integrate parts with their relations in the mental representation of an object category.

What is the mechanism whereby connectedness affects part-relation integration in object category learning? One intuitive possibility is that the human cognitive apparatus attempts to define object equivalence classes on single objects (rather than clusters of objects in the visual field) and that connectedness is an important cue for deciding whether a collection of parts forms a single object (Saiki \& Hummel, 1998) - that is, for deciding whether a collection of parts is a reasonable candidate for defining an object equivalence class. This account is consistent with the perceptual literature on part-relation integration (Palmer \& Rock, 1994; Saiki \& Hummel, 1998 ) and feature-relation integration (Baylis \& Driver, 1993), and it suggests that the formation of part-relation structures in long-term memory (i.e., the formation of object equivalence classes) obeys constraints similar to those governing the formation of part-relation structures in shape perception. As such, it underscores the possibility that the memory representations underlying categorization and category learning are both more perceptual and more structured than has traditionally been assumed (Barsalou, 1993).

Alternatively, all stimuli may be represented by abstract symbols for parts, relations, and their conjunctions, but connectedness may affect the weighting of partrelation conjunctions (with higher weights for connected stimuli than for nonconnected stimuli). This idea is consistent with traditional categorization models, in which objects are represented in terms of abstract dimensions (Nosofsky, 1986) or features (Gluck \& Bower, 1988). In such models, perceptual constraints are captured by the metric structure of the psychological space (e.g., by expanding or shrinking various dimensions; however, see Holyoak \& Hummel, in press, and Hummel, in press, for discussions of serious limitations of this approach to coding object structure). Although it remains to be seen which alternative is more plausible, it is clear that per- ceptual processing has substantial effects on the memory representations mediating object categorization. The details of the mechanisms linking connectedness and category learning--including the role of attention-are a question for future research.

\section{Object Categorization and \\ Object Memory Systems}

In this study, we used a variety of categorization tasks to examine the effects of connectedness on part-relation integration in object memory. We do not assume that the tasks we used (and the findings we observed) reflect the properties of object memory in general. Rather, we used these tasks specifically to explore the object memory system(s) responsible for categorization and recognition. Recent studies have shown evidence of different memory systems subserving different aspects of visual cognition. For example, Marsolek (1995) reported evidence of two different visual form systems: an abstract visual form system and a specific visual form system. Srinivas (1995, 1996; also see Cave, Bost, \& Cobb, 1996) found systematic dissociations between implicit and explicit memory in terms of their sensitivity to various properties of a stimulus display. Specifically, whereas implicit memory seems selective for properties that are inherent to the object itself (such as aspects of its shape, etc.), explicit memory seems to store many aspects of the specific episode in which an object is experienced (see also Cooper, Biederman, \& Hummel, 1992). The memory systems tapped by categorization tasks differ from those tapped by more explicit memory tasks (Knowlton \& Squire, 1996; Knowlton, Squire, \& Gluck, 1994). It remains to be seen which system or systems are responsible for the connectedness effects reported here, but it seems likely that they reflect the operation of those responsible for recognition and categorization, rather than those responsible for memory for specific episodes.

\section{REFERENCES}

AshbY, F. G., \& MAdDox, W. T. (1994). A response time theory of separability and integrality in speeded classification. Journal of Mathematical Psychology, 38, 423-466.

BARRETT, S. E., \& ShEPP, B. E. (1988). Developmental changes in attentional skills: The effect of irrelevant variations on encoding and response selection. Journal of Experimental Child Psychology, 45, 382-399.

BarSALOU, L. W. (1993). Flexibility, structure, and linguistic vagary in concepts: Manifestations of a compositional system of perceptual symbols. In A. F. Collins, S. E. Gathercole, M. A. Conway, \& P. E. Morris (Eds.), Theories of memory (pp. 29-101). Hillsdale, NJ: Erlbaum.

BayLis, G. C., \& Driver, J. (1993). Visual attention and objects: Evidence for hierarchical coding of location. Journal of Experimental Psychology: Human Perception \& Performance, 19, 451-470.

BaYLIS, G. C., \& Driver, J. (1995). One-sided edge assignment in vision: 1. Figure-ground segmentation and attention to objects. Current Directions in Psychological Science, 4, 140-146.

BIEDERMAN. I. (1987). Recognition-by-components: A theory of human image understanding. Psychological Review, 94, 115-147. CAVE, C. B., Bost, P. R., \& CoBB, R. E. (1996). Effects of color and pat- 
tern on implicit and explicit picture memory. Journal of Experimental Psychology: Learning, Memory, \& Cognition, 22, 639-653.

CoOper, E. E., Biederman, I., \& Hummel, J. E. (1992). Metric invariance in object recognition: A review and further evidence. Canadian Journal of Psychology, 46, 191-214.

Donnelley, N., Humphreys, G. W., \& Riddoch, M. J. (1991). Parallel computation of primitive shape descriptions. Journal of Experimental Psychology: Human Perception \& Performance, 17, 561 570.

Elder, J., \& ZuCKER, S. (1993). The effect of contour closure on the rapid discrimination of two-dimensional shapes. Vision Research, 33, 981-991.

ENNS, J. T. (1992). Sensitivity of early human vision to 3-D orientation in line-drawings. Canadian Journal of Psychology, 46, 143-169.

FELfoldy, G. L. (1974), Repetition effects in choice reaction time to multidimensional stimuli. Perception \& Psychophysics, 15, 453459.

FODOR, J. A., \& PYLYSHYN, Z. W. (1988). Connectionism and cognitive architecture: A critical analysis. In S. Pinker \& J. Mehler (Eds.) Connections and symbols (pp. 3-71). Cambridge, MA: MIT Press.

GARNER, W. R. (1974). The processing of information and structure. Hillsdale, NJ: Erlbaum.

GluCK, M. A., \& Bower, G. H. (1988). From conditioning to category learning: An adaptive network model. Journal of Experimental $P$ sychology: General, 117, 227-247.

Grau, J. W., \& Kemler Nelson, D. G. (1988). The distinction between integral and separable dimensions: Evidence for the integrality of pitch and loudness. Journal of Experimental Psychology: General, 117, 347-370.

Holyoak, K. J., \& Hummel, J. E. (in press). The proper treatment of symbols in connectionism. In E. Dietrich \& A. Markman (Eds.), Cognitive dynamics: Conceptual change in humans and machines. Cambridge, MA: MIT Press.

Hummel, J. E. (in press). Where view-based theories break down: The role of structure in shape perception and object recognition. In E. Dietrich \& A. Markman (Eds.), Cognitive dynamics: Conceptual change in humans and machines. Cambridge, MA: MIT Press.

Hummel, J. E., \& Biederman, I. (1992). Dynamic binding in a neural network for shape recognition. Psychological Review, 99, 480-517.

Hummel, J. E., \& Holyoak, K. J. (1997). Distributed representations of structure: A theory of analogical access and mapping. Psychological Review, 104, 427-466.

Hummel, J. E., \& SAIKI, J. (1993). Rapid unsupervised learning of object structural descriptions. In Proceedings of the 15th Annual Conference of the Cognitive Science Society (pp. 569-574). Hillsdale, NJ: Erlbaum.

Hummel, J. E., \& STANkiewicz, B. J. (1996). An architecture for rapid, hierarchical structural description. In T. Inui \& J. McClelland (Eds.), Attention and performance XVI: Information integration in perception and communication (pp. 93-121). Cambridge, MA: MIT Press.

Humphreys, G. V., \& BrucE, V. (1989). Visual cognition. London: Erlbaum

Hunt, S. (1993). MacProbe [Computer program]. Los Angeles: Aristometrics.

Kemler Nelson, D. G. (1993). Processing integral dimensions: The whole view. Journal of Experimental Psychology: Human Perception \& Performance, 19, 1105-1113.
Kimchi, R., \& Palmer, S. E. (1985). Separability and integrality of global and local levels of hierarchical patterns. Journal of Experimental Psychology: Human Perception \& Performance, 11, 673688.

KNOWLton, B. J., \& SQuire, L. R. (1996). Artificial grammar learning depends on implicit acquisition of both abstract and exemplarspecific information. Journal of Experimental Psychology: Learning, Memory, \& Cognition, 22, 169-181.

Knowlton, B. J., Squire, L. R., \& Gluck, M. A. (1994). Probabilistic classification learning in amnesia. Learning \& Memory, 1, 106120.

LOCKHEAD, G. R. (1966). Effects of dimensional redundancy on visual discrimination. Journal of Experimental Psychology, 72, 95-104.

LowE, D. G. (1987). The viewpoint consistency constraint. International Journal of Computer Vision, 1, 57-72.

MARR, D., \& NishihaRA, H. K. (1978). Representation and recognition of the spatial organization of three-dimensional shapes. Proceedings of the Royal Society of London: Series B, 200, 269-294.

MarsoleK, C. J. (1995). Abstract visual-form representations in the left cerebral hemisphere. Journal of Experimental Psychology: Human Perception \& Performance, 21, 375-386

NosofSKy, R. M. (1986). Attention, similarity, and the identificationcategorization relationship. Journal of Experimental Psychology: General, 115, 39-57.

PAlmer, S. E. (1992). Common region: A new principle of perceptual grouping. Cognitive Psychology, 24, 436-447.

PALMER, S. E., \& RoCK, I. (1994). Rethinking perceptual organization: The role of uniform connectedness. Psychonomic Bulletin \& Review, 1, 29-55.

Peterson, M. A., \& Gibson, B. S. (1994). Must figure-ground organization precede object recognition? An assumption in peril. Psychological Science, 5, 253-259.

Pomerantz, J. R., \& Pristach, E. A. (1989). Emergent features, attention, and perceptual glue in visual form perception. Journal of Experimental Psychology: Human Perception \& Performance, 15, 635-649.

Rosch, E., Mervis, C. B., Gray, W. D., Johnson, D. M., \& BoyesBraem, P. (1976). Basic objects in natural categories. Cognitive Psychology, 8, 382-439.

SAIKI, J., \& Hummel, J. E. (1996). Attribute conjunctions and the part configuration advantage in object category learning. Journal of Experimental Psychology: Learning, Memory \& Cognition, 22, 10021019.

Saiki, J., \& Hummel, J. E. (1998). Connectedness and the partrelation integration in object perception. Journal of Experimental Psychology: Human Perception \& Performance, 24, 227-251.

Shepp, B. E., \& Barrett, S. E. (1991). The development of perceived structure and attention: Evidence from divided and selective attention tasks. Journal of Experimental Child Psychology, 51, 434-458

SRINIVAS, K. (1995). The representation of rotated objects in explicit and implicit memory. Journal of Experimental Psychology: Learning, Memory, \& Cognition, 21, 1019-1036.

SRINIVAS, K. (1996). Contrast and illumination effects on explicit and implicit measures of memory. Journal of Experimental Psychology: Learning, Memory, \& Cognition, 22, 1123-1135.

(Manuscript received March 20, 1997; revision accepted for publication September 15, 1997.) 